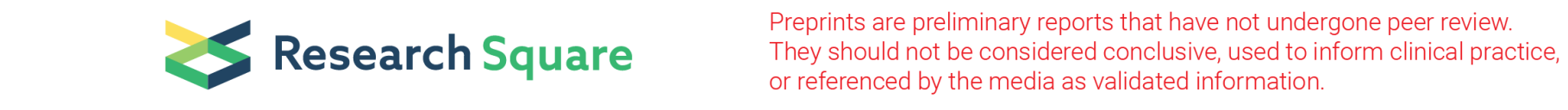

\title{
Perturbation of PI3K/Akt Signaling Affected Autophagy Modulation in Dystrophin-deficient Myoblasts
}

\author{
Muhammad Dain Yazid ( $\sim$ dain@ukm.edu.my ) \\ Universiti Kebangsaan Malaysia https://orcid.org/0000-0001-6162-4405 \\ Hung-Chih Chen \\ Academia Sinica
}

\section{Research}

Keywords: Myoblasts, Dystrophin, PI3K/Akt signalling, Autophagy, Duchenne Muscular Dystrophy

Posted Date: November 30th, 2020

DOI: https://doi.org/10.21203/rs.3.rs-113469/v1

License: @ (i) This work is licensed under a Creative Commons Attribution 4.0 International License. Read Full License 


\section{Abstract}

Background: The absence of dystrophin has gave a massive impact on myotube development in Muscular Dystrophy pathogenesis. One of the conserved signaling pathways involved in skeletal muscle differentiation is the PI3K/Akt/mTOR pathway that plays a vital role in autophagy regulation. To further understand and establish targeted therapy in dystrophindeficient myoblasts, protein expression profiling has been determined which provides information on perturbed autophagy modulation and activation.

Methods: In this study, a dystrophin-deficient myoblast cell line established from the skeletal muscle of a dystrophic (mdx) mouse was used as a model. The dfd13 (dystrophin-deficient) and C2C12 (non-dystrophic) myoblasts were cultured in low mitogen conditions for 10 days to induce differentiation. The cells were subjected to total protein extraction prior to Western blotting assay technique. Protein sub-fractionation has been conducted to determine protein localization. The live-cell analysis of autophagy assay was done using a flow cytometer.

Results: In our culture system, the dfd13 myoblasts did not achieve terminal differentiation. PTEN expression was profoundly increased in dfd13 myoblasts throughout the differentiation day subsequently indicates perturbation of PI3K/Akt/mTOR regulation. In addition, rictor-mTORC2 was also found inactivated in this event. This occurrence has caused FoxO3 misregulation leads to higher activation of autophagy-related genes in dfd13 myoblasts. Autophagosome formation was increased as LC3B-I/II showed accumulation upon differentiation. However, the ratio of LC3B lipidation and autophagic flux were shown decreased which exhibited dystrophic features.

Conclusion: perturbation of the PTEN-PI3K/Akt pathway triggers excessive autophagosome formation and subsequently reduced autophagic flux within dystrophin-deficient myoblasts where these findings are of importance to understand Duchenne Muscular Dystrophy (DMD) patients. We believe that some manipulation within its regulatory signaling reported in this study could help restore muscle homeostasis and attenuate disease progression.

\section{Background}

Duchenne muscular dystrophy (DMD) is an inherited muscle disease caused by deletion, duplication or a point mutation of the $d m d$ gene (dystrophin) on the human $\mathrm{X}$ chromosome (1). DMD is characterized by progressive skeletal muscle weakness, defects in muscle proteins, and the death of muscle tissue (2). The absence of dystrophin has a massive impact on myoblast structure. The destabilization of the plasma membrane in dystrophin-deficient myoblasts from an mdx mouse (DMD model) affects transmembrane protein stability, as well as the protein-anchored cytoplasmic layer of the cell membrane thus changing signaling $(3,4)$. One of the conserved signaling pathways for skeletal muscle differentiation is the PI3K/Akt/mTOR pathway. The PTEN-PI3K/Akt/mTOR signaling pathway regulates cell proliferation, differentiation, autophagy, and apoptosis (5-7). PTEN acts as a lipid phosphatase and hydrolyses phosphates in the 3 ' position of phosphoinositides. Previous studies have reported that PTEN mRNA is elevated in the $m d x$ muscles $(8,9)$. The elevation of PTEN expression and its activity has also been shown to contribute to profound PI3K/Akt signaling pathway deregulation in dystrophin-deficient dog muscle (10).

Similarly, PTEN has been reported to contribute to the DMD phenotype. A study by Feron et al. (2009) on the Golden Retriever muscular dystrophy (GRMD) dog demonstrated that PTEN was present at high levels, which led to a reduction of Akt1, GSK3 $\beta$ and p70S6K, also, ERK1/2 displayed decreased phosphorylation levels in GRMD dog muscle. The GRMD dog is characterized by rapidly progressive clinical dysfunction, severe muscle weakness, and displays a disease progression that is more similar to human DMD compared to the $m d x$ mouse (10).

Akt plays a major role in cell signaling and its impairment leads to various defects in downstream protein signaling, including cell proliferation, differentiation, and apoptosis, autophagy, and protein synthesis perturbation. Activation of Akt is higher in the $m d x$ mouse $(8,11)$ as well as in DMD patients $(12)$. Akt has also been reported to be highly activated and associated with aintegrin in the $m d x / u t r^{-/}$mouse model (13). Boppart et al. (2011) demonstrated that a-integrin transgenic $m d x / u t r^{--}$mice (a7ßX2-mdx/utr-/-) showed increased phosphorylation of Akt at Ser473, indicating that a-integrin expression is connected to 
Akt. The $m d x / u t r /-$ mouse lacks both dystrophin and utrophin and develops a severe pathology that closely resembles that seen in DMD (13). That DMD animal model was found compensated for the dystrophin loss and both dystrophin and utrophin loss, respectively. Therefore, Akt was found activated in both models as it was found to be connected to a-integrin.

Autophagy is the process of engulfment of cargo into the double lipid formation known as an autophagosome, which eventually fuses with a lysosome and is degraded (14). Autophagic activity is rapidly increased in cells under stress conditions and nutrient deprivation to maintain cell homeostasis (15). Generally, defective autophagy exhibits a dual response; at high levels, it causes muscle atrophy, whilst at low levels, it contributes to muscle degeneration. High levels of autophagy result in excessive protein degradation due to high levels of autophagy gene activation (15). In this state inactivation of Akt inhibits the activation of autophagy-related genes indirectly via mTOR and/or directly phosphorylates the FoxO3a transcription factor (16). This event leads to reduced muscle mass and muscle wasting. In contrast, low-level autophagy is defined as low autophagy activity which causes an accumulation of dysfunction and unused organelles, i.e. mitochondria, as well as unfolded protein within skeletal muscle. This condition leads to an altered muscle structure with prominent myonuclei centralization and fusion abnormalities, which eventually weakens the muscle leading to the dystrophic phenotype (17). Therefore, maintaining autophagy at the appropriate level is crucial within skeletal muscle.

Autophagy is defective in $m d x$ mice (18) and DMD humans (19). Restoration of beclin1 levels in Col6a ${ }^{\%}$ animals and longterm exposure to a low-protein diet $(11,15)$ can reactivate autophagy and partly ameliorate the dystrophic features/phenotype. Col6a $1^{-1-}$ animals display impairment of basal autophagy, which determines the persistence of dysfunctional organelles in muscle fibers leading to muscle degeneration (15). Treatment with a long-term low-protein diet can reactivate autophagy by normalized Akt activation, thus increasing LC3B conversion and up-regulating autophagy-related genes (19).

Over a decade, many researchers have reported on impairment of Igf-1 pathway and its downstream protein regulation in muscular dystrophy. However, to further understand the impaired regulation of autophagy in dystrophin-deficient myoblast, the protein profiling of this pathway has been determined which sequentially provides information on autophagy modulation and activation. In the beginning, we reported the capacity of dystrophin-deficient myoblasts differentiation via analysis of myotube formation and the expression of specific myosin heavy chain as a terminal differentiation marker. Furthermore, we showed the elevation of PTEN perturbed PI3K/Akt/mTOR signaling thus excessively activate autophagy-related genes via FoxO3 but subsequently showed a reduction of autophagic flux in dystrophin-deficient myoblast. Overview of this event as illustrated at the end of this article.

\section{Materials And Methods}

\section{Cell culture and differentiation}

The $\mathrm{C} 2 \mathrm{C} 12$ myoblast cell line was used in this study and was established from an adult mouse myoblast C2 cell line derived from the thigh muscle of 2-month-old mice (20). The dfd13 cell line was derived from a 5-week-old mdx mouse $(21,22)$. Mouse embryonic fibroblast (MEF) cells were a gift from Adil Rashid, University of Birmingham, and were used as a control for the phosphorylation of Akt at threonine-308. Both myoblasts were maintained in growth medium (GM) consist of Dulbecco's Modified Eagles Medium (DMEM) (Invitrogen Ltd, UK) supplemented with 10\% Fetal Bovine Serum (FBS) (Sigma-Aldrich, UK), 1\% penicillin/streptomycin (Sigma-Aldrich, UK) and 1\% L-Glutamine (Sigma-Aldrich, UK) before plated for differentiation. To differentiate myoblasts, GM was replaced with differentiation medium (DM) which is DMEM supplemented with $2 \%$ Horse Serum (Sigma-Aldrich, UK), 1\% penicillin/streptomycin (Sigma-Aldrich, UK), and 1\% L-Glutamine and cultured for 10 days.

\section{Total protein extraction}

Protein was extracted from cells cultured in a $10 \mathrm{~cm}$ dish. The medium was removed and the cells washed twice with PBS before adding approximately $300 \mu \mathrm{L}$ of lysis buffer $(0.5 \%$ Triton $\mathrm{X}-100,0.5 \%$ deoxycholic acid; $0.5 \mathrm{M} \mathrm{NaCl} ; 0.02 \mathrm{M}$ Tris; $0.01 \mathrm{M}$ EDTA, pH 7.5) containing a protease inhibitor (complete ULTRA tablets, EDTA-free, protease inhibitor cocktail, Roche, UK). The 
cells were scraped and collected into a tube before being subjected to centrifugation at $14000 \mathrm{rpm}$ for 15 minutes at $4^{\circ} \mathrm{C}$. The pellet was discarded and the supernatant transferred to a new tube and stored at $-20^{\circ} \mathrm{C}$.

\section{Protein sub-fractionation}

The rapid efficient and practical (REAP) protocol was used for protein sub-fractionation (23). All reagents were chilled and kept on ice at all times. The medium was removed and the cells were washed three times with PBS before adding approximately 1 $\mathrm{mL}$ of ice-cold PBS. The cells were scraped and collected into a new tube and pop-spun for 10 seconds and the supernatant discarded. The pellet was resuspended in $900 \mu \mathrm{L}$ of ice-cold PBS containing $0.1 \%$ nonyl-phenoxypolyethoxylethanol (NP-40) and approximately $300 \mu \mathrm{L}$ was removed to a new tube labeled as whole cell lysate $(\mathrm{WCL})$. The remaining cells were pop-spun for 10 seconds and approximately $300 \mu \mathrm{L}$ was transferred to a new tube labeled as the cytosolic fraction, while the remainder of the supernatant was discarded. The pellet was washed with $1 \mathrm{~mL}$ of PBS containing $0.1 \% \mathrm{NP}-40$ ) and pop-spun for 10 seconds; this step was repeated 2 to 5 times to eliminate the cytosolic fraction. After washing was completed this was considered to be the nuclear fraction. The cytosolic fraction was also pop-spun 3 times to separate the remaining nuclear fraction. Both the WCL and nuclear fraction were sonicated three times for 10 seconds and boiled for 1 minute.

\section{Western blotting}

Equal protein loading was prepared before resuspending with $2 \times$ Laemmli sample buffer. The mixture was collected by short spin centrifugation and boiled for 5 minutes before loaded into SDS-polyacrylamide gel electrophoresis. Lysate was separated on SDS-polyacrylamide gel electrophoresis $(6,8$, or $15 \%$ acrylamide) with $200 \mathrm{~V}$ for 1 hour. The separated protein was transferred onto the nitrocellulose membrane using the Transblot Turbo ${ }^{\circledR}$ Transfer System (BioRad, UK). The membranes were blocked in $5 \%$ reduced-fat milk for 1 hour before incubate with primary antibodies. The antibodies are Fast-MyHC, a-tubulin, SSRP-1 (1:1000) and Desmin, (1:2000), from Sigma-Aldrich, UK. PTEN (1:1000) (a gift from Dr. Zubair Ahmed, Medical School, University of Birmingham). PI3K, phospho-PI3K (Tyr458), Akt, phospho-Akt (Ser473), mTOR, phospho-mTOR (Ser2448), p70S6 Kinase, phospho-p70S6 Kinase, Rictor, phospho-rictor (Thr1135) and FoxO3a (1:1000) from Cell Signalling Technology, UK. Beclin1, Atg5, Atg7, and LC3B (1:1000) (a gift from Dr. Melissa Grant, School of Dentistry, University of Birmingham).

\section{Immunofluorescence}

The coverslips were acid-etched for 5 minutes with nitric acid. This will help myoblasts firmly attached to the coverslips before immunofluorescence. It has been sent for sterilization before myoblasts were seeded onto it. The myoblasts/myotubes were fixed using 4\% PFA. After fixation cells were permeabilized with $0.25 \%$ Triton X-100 for 5 minutes to allow the antibody to bind specifically. The cells were then washed three times for 5 minutes with PBS before blocking with $5 \%$ BSA at room temperature for 30 minutes. The blocking solution was removed and the cells incubated with the primary antibody Fast-MyHC at $4^{\circ} \mathrm{C}$ overnight. The primary antibody was removed the next day and the cells washed before probed using a secondary antibody (biotinylated) for 1 hour at room temperature. After washing, the cells were incubated with streptavidin-Texas red (1:1000) at room temperature for 1 hour in the dark. The cells were then counterstained with DAPI (1:100) and mounted onto slides using DakoCytomation. The slides were wrapped in aluminum foil and stored at $4^{\circ} \mathrm{C}$.

\section{Autophagy assay: cells labeling}

An autophagy assay was performed using a CYTO-ID Autophagy Detection Kit (Enzo Life Science, Switzerland) and $1 \mathrm{x}$ $10^{5} / \mathrm{mL}$ myoblasts were added to every well in a 24 -well plate and maintained via standard tissue culture practice. Cells were grown in a humidified incubator at $37^{\circ} \mathrm{C}$ and $5 \% \mathrm{CO}_{2}$; cells were not allowed to become over-crowded and the density should not exceed $1 \times 10^{6} / \mathrm{mL}$. In this experiment, $60 \mathrm{mM}$ of chloroquine was used as a positive control, while unstained cells were utilized as a negative control. Myoblasts were treated with chloroquine for 4 hours in a humidified incubator at $37^{\circ} \mathrm{C}$ and $5 \%$ 
$\mathrm{CO}_{2}$. At the end of the treatment, cells were trypsinized and collected into a fresh $1.5 \mathrm{~mL}$ microtube. Cells were subjected to centrifugation for 5 minutes at $1000 \mathrm{rpm}$ at room temperature and washed by re-suspending in $1 \mathrm{x}$ assay buffer and the centrifugation step was repeated. Each cell pellet was then re-suspended in $250 \mu \mathrm{L}$ of $1 \mathrm{x}$ assay buffer. Approximately $250 \mu \mathrm{L}$ of the diluted CYTO-ID Green stain solution was added to all the cells except the negative control and incubated for 30 minutes at room temperature in the dark. The cells were collected by centrifugation, washed with $1 \mathrm{x}$ assay buffer, and the pelleted cells resuspended in $500 \mu \mathrm{L}$ of $1 \mathrm{x}$ assay buffer. The samples were analyzed using the green (FL1) channel of a flow cytometer.

\section{Flow cytometer}

Experiments were performed using a FACS Analyser CyAn B (Beckman Coulter, USA) at the Institute of Biomedical Research, University of Birmingham. Samples were run using Summit V4.3 software and all data were saved in the FCS format developed by the Society of Analytical Cytology, thereby allowing further analysis using other software packages. Data were analyzed using the software FCS Express 6 Plus (De Novo Software, USA) for three parameters; side scatter (SS), forward scatter (FS), and Fluorescein Isothiocyanate (FITC). Gating was performed based on the size and complexity of the myoblasts as illustrated in density plots. Gated data were further analyzed to count myoblasts labeled with FITC and the results presented in a histogram format.

\section{Statistical analysis}

All Western blots and flow cytometry were repeated at least three times for each experiment. Statistical analyses were carried out using the student t-test (Microsoft Excel) and one-way ANOVA. The differences were considered significant when $p<0.05$ and $p<0.01$.

\section{Results}

\section{Dystrophin-deficient myoblasts do not achieve terminal differentiation}

In vitro differentiation of myoblasts can be induced in culture through the use of a low mitogen medium (differentiation medium; DM) for a few days. In this study, both C2C12 (non-dystrophic) and dfd13 (dystrophin-deficient) myoblasts were cultured in low mitogen medium (differentiation medium; DM) for 10 days before determining their terminal differentiation capacity. Immunofluorescence analyses (Fig 1A-B) showed both non-differentiated and differentiated myoblasts. Multinucleated myotube formation can be seen on day 10 of differentiation in C2C12 (A) myoblasts but is hardly/rarely found in dfd13 myoblasts where less multinucleated cells were observed (Fig 1B). This indicates that dfd13 differentiation capacity is impaired; however, the differentiating dfd13 myoblasts can be seen to be aligned and seem to be ready for cell fusion to become myotubes.

Myotube formation was determined by counting the myonuclei present in a myotube that expressed MyHC (fast-type II, F$\mathrm{MyHC}$ ) and then normalized against total nuclei. F-MyHC, also called 'fast-twitch' fibers, were chosen as the terminal differentiation marker based on the existence of a spectrum of fiber types, with type II being the most developed form of myosin: type $1 \leftrightarrow 1 / 2 \mathrm{~A} \leftrightarrow 2 \mathrm{~A} \leftrightarrow 2 \mathrm{~A} / 2 \mathrm{X} \leftrightarrow 2 \mathrm{X} \leftrightarrow 2 \mathrm{X} / 2 \mathrm{~B} \leftrightarrow 2 \mathrm{~B}(24)$. The number of myonuclei in $\mathrm{C} 2 \mathrm{C} 12$ myotubes was 9 -fold higher compared to myonuclei in differentiating dfd13 myoblasts $(p<0.01)$. The percentage of myonuclei per total nuclei was $80.2 \%$ in $\mathrm{C} 2 \mathrm{C} 12$ myotubes and $8.2 \% 6$ in differentiating dfd13 myoblasts (Fig 1C).

Immunoblotting for F-MyHC, pan-myosin, and desmin was performed on days 4, 7, and 10 of differentiation (Fig 1D). Generally, F-MyHC expression was increased upon differentiation in $\mathrm{C} 2 \mathrm{C} 12$ myoblasts but none of the differentiating dfd13 myoblasts showed any expression (Fig 1E). However, pan-myosin was expressed at day 10 in dfd13 myoblasts (Fig 1F), and there was a significant difference in expression $(\mathrm{p}<0.01)$ when compared to $\mathrm{C} 2 \mathrm{C} 12$ myoblasts. Desmin expression in both myoblasts was also examined and was found to be drastically increased upon differentiation, in C2C12 myoblasts. Desmin was used as an intermediate differentiation marker and is a muscle specific type II intermediate filament that integrates the sarcolemma, Z-disk 
and nuclear membrane in myoblasts. In differentiating dfd13 myoblasts, desmin expression gradually increased over the 10 days and was significantly higher $(p<0.01)$ in comparison to levels in $\mathrm{C} 2 \mathrm{C} 12$ myotubes (day 10) (Fig 1G).

\section{PTEN-PI3K regulation is perturbed in differentiating dystrophin-deficient myoblasts}

Generally, PTEN expression was increased upon differentiation in both types of myoblasts. However, the accumulation of PTEN was found to be higher in differentiating dfd13 myoblasts compared to $\mathrm{C} 2 \mathrm{C} 12$ myoblasts (Fig 2A). The accumulation of PTEN throughout differentiation was not significant in $\mathrm{C} 2 \mathrm{C} 12$ myoblasts but there were significant increases on day $7(p<0.05)$ and day $10(p<0.05)$ when compared to non-differentiated cells. Densitometry analysis showed that PTEN expression was significantly higher $(\mathrm{p}<0.05)$ in dfd13 myoblasts compared to $\mathrm{C} 2 \mathrm{C} 12$ in the non-differentiated stage. The differentiating dfd13 myoblasts were also showed significantly higher compared to the differentiating C2C12 myoblasts on days 4, 7, and 10 (Fig 2B).

PTEN has a negative effect on PI3K, as it concomitantly affects PI3K regulation. PI3K expression and its activity in both types of myoblasts were therefore examined. Generally, total PI3K expression was increased in $\mathrm{dfd} 13$ myoblasts throughout the differentiation period. However, significant accumulation of PI3K could be seen by day $7(p<0.01)$ and day $10(p<0.01)$ when compared to the non-differentiated dfd13 myoblasts. There was no significant difference in total PI3K expression in $\mathrm{C} 2 \mathrm{C} 12$ myoblasts throughout the differentiation period compared to non-differentiated myoblasts. Densitometry analysis showed that there were significant differences in total PI3K expression in dfd13 myoblasts on day $4(p<0.05)$, day 7 ( $p<0.01)$, and day 10 when compared to differentiating $\mathrm{C} 2 \mathrm{C} 12$ myoblasts (Fig 2C). As illustrated in Figure 2D, densitometry analysis on PI3K activity was higher in dfd13 myoblasts at the non-differentiated stage, day 4, and reduced at day 10 of differentiation when compared to $\mathrm{C} 2 \mathrm{C} 12$ myoblasts.

\section{Akt is less activated in dystrophin-deficient myoblasts}

As PTEN is highly expressed in dfd13 myoblasts, it is predicted that Akt is not/less activated, as PTEN has previously been reported to modulate Akt activation in rhabdomyosarcomas cells; skeletal muscle cancer (25). In this study phosphorylation of Akt was not detected at Ser473 or Thr308 in dfd13 myoblasts during differentiation (Fig 3A). It has been reported that phosphorylation of Akt by PDK1 at Thr308 partially activates Akt while full activation requires phosphorylation of Ser473 which can be catalyzed by multiple proteins including Rictor-mTORC2 (26). This activation is responsible for myoblast proliferation as well as differentiation.

As depicted in Figure 3B, total Akt expression increased in both types of myoblast during differentiation, with slightly higher levels present in dfd13 myoblasts (no significant difference). Densitometry analysis of Akt activation via phosphorylation at Ser473 (Fig 3C) showed a significant difference when compared to C2C12 myoblast at all stages (non-differentiated. There was also a significant difference in Akt activation at Ser473 in C2C12 myoblasts when compared to the non-differentiated stage.

\section{Rictor-mTORC2 is less activated in dystrophin-deficient myoblasts}

A previous study reported that mTORC2 is essential for terminal myogenic differentiation (27). It was also reported that it participates in actin cytoskeleton arrangements (26), which might be involved in dystrophin functionality in myoblasts. Therefore, it was hypothesized that Akt inactivation is affected by rictor, a subunit of mTORC2, upstream of Akt, and responsible for Ser473 phosphorylation. However, the upstream protein that regulates rictor-mTORC2 remains unclear. In this study, both total rictor and mTOR expression was examined as well as its activation. However, it was impossible to classify the specific complexes of mTOR activity as phosphorylation at Ser2448 can cause the binding to both raptor and rictor (28). 
As depicted in Figure 4A, immunoblot analysis of total rictor, mTOR and their phosphorylated forms was performed.

Phosphorylated-rictor at Thr1135 was virtually not detected in dfd13 myoblasts throughout the differentiation period. However, rictor expression was increased upon differentiation in both types of myoblasts. Densitometry analysis showed that there was no significant difference in its accumulation in the two types of myoblasts Fig 4B). Rictor expression only showed a significant increase $(p<0.05)$ in dfd13 myoblasts at day 10 compared to non-differentiated dfd13 myoblasts. Densitometry analysis of rictor activation by phosphorylation at Thr1135 (Fig 4C) showed a significant difference in C2C12 myoblasts compared to dfd13 myoblasts at all stages.

Figure 4D shows that total mTOR expression was increased in dfd13 myoblasts during differentiation but remained the same in C2C12 myoblasts. A significant accumulation was seen in dfd13 myoblasts on days 4, 7, and 10 when compared to C2C12 myoblasts. mTOR activity was significantly higher in non-differentiated dfd13 myoblasts when compared to non-differentiated $\mathrm{C} 2 \mathrm{C} 12$ myoblasts. The activity showed a significant reduction upon differentiation in dfd13 myoblasts while $\mathrm{C} 2 \mathrm{C} 12$ myoblasts showed a significant accumulation when compared to dfd13 myoblasts on days 4, 7, and 10 (Fig 4E). Expression and activation of mTOR were considered to represent endogenous levels of mTOR within both complexes i.e. mTORC1 or mTORC2, where distinct activation occurred. Phosphorylation of mTOR at Ser2448 makes it a major target for p70S6 kinase activation and is also an important event for raptor and rictor binding (28).

\section{Fox03 expression is highly increased and predominantly localized in the nucleus of differentiating dystrophin-deficient myoblasts}

Inactivation of Akt affected another downstream protein, FoxO3 (Forkhead box 03), which is a target protein of Akt. FoxO3 is a transcription factor responsible for the activation of autophagy genes involved in the autophagy machinery. A previous study showed that FoxO3 controls autophagy in skeletal muscle in vivo and induced multiple autophagy genes, including LC3B transcription in skeletal muscle (29). In this study, FoxO3 expression in myoblasts was examined.

Immunoblot analyses (Fig 5A) showed that FoxO3 expression was increased in both types of myoblasts during differentiation. However, there was only a slight increase in FoxO3 in $\mathrm{C} 2 \mathrm{C} 12$ myoblasts on day 4, and it remained at the same level until day 10. In non-differentiated dfd13 myoblasts FoxO3 levels were found to be significantly lower $(\mathrm{p}<0.01)$ compared to $\mathrm{C} 2 \mathrm{C} 12$ myoblasts. FoxO3 then increased throughout the differentiation period and there was a significant difference in dfd13 myoblasts on day 10 compared to C2C12 myoblasts (Fig 5B).

Akt plays a role in inhibiting FoxO3 through phosphorylation at Thr24, Ser256, and Ser319, which leads to nuclear exclusion and activation. As Akt is inactivated in dfd13 myoblasts, it is thought that FoxO3 is not phosphorylated. Therefore, the nonphosphorylated FoxO3 is translocated to the nucleus and activates the gene involved in autophagy. Akt represses FoxO3 via phosphorylation resulting in nuclear exclusion. As Akt is not activated in dfd13 myoblasts, it was speculated that unphosphorylated-FoxO3 translocates into the nucleus and binds to the promoter, thus up-regulating autophagy-related genes such as LC3B, Atg5, and Atg7. Therefore, FoxO3 expression and localization needed to be examined within subcellular fractions, i.e. the nucleus and cytoplasm.

FoxO3 was found to be localized more to the nucleus of dfd13 myoblasts (Fig 5C), with approximately 81.6\% of Fox03 present in the nucleus of dfd13 myoblasts compared to only $~ 47.6 \%$ in $\mathrm{C} 2 \mathrm{C} 12$ myoblasts during the undifferentiated stage.

Surprisingly, on day 10 of differentiation, levels in the nucleus had accumulated in both types of myoblast; $\mathrm{C} 2 \mathrm{C} 12 \sim 78.2 \%$, and dfd13 94.4\% (Fig 5D).

\section{Autophagy related proteins are highly increased in differentiating dystrophin-deficient myoblasts}


It has been shown that FoxO3 is highly expressed and primarily localized to the nucleus of differentiating dfd13 myoblasts. Within the nucleus, FoxO3 acts as a transcriptional activator that can recognize and bind to DNA sequences resulting in the activation of genes involved in autophagy, such as those for Beclin1 and Atgs (29). It was hypothesized that autophagy is highly activated in differentiating dystrophin-deficient myoblasts.

Figure 6 presents immunoblot and densitometry analyses of autophagy-related proteins involved in autophagosome formation. Beclin1 expression was slightly increased in $\mathrm{C} 2 \mathrm{C} 12$ myoblasts and highly increased in dfd13 myoblasts upon differentiation (Fig 6A). Densitometry analysis of Beclin1 expression showed significant accumulation on days 4, 7, and 10 in dfd13 myoblasts compared to C2C12 myoblasts (Fig 6B).

Atg5 was found to be not/less expressed in $\mathrm{C} 2 \mathrm{C} 12$ myoblasts (C). It can be seen that Atg5 expression was increased upon differentiation in dfd13 myoblasts, and expression was significantly increased on days 4, 7, and 10 compared to the nondifferentiated stage. Densitometry analysis also showed significant differences $(p<0.01)$ for dfd13 myoblasts at every time point compared to $\mathrm{C} 2 \mathrm{C} 12$ myoblasts (Fig 6C).

Densitometry analysis of Atg7 expression in dfd13 myoblasts showed its accumulation throughout the differentiation period; however, there was no significant difference in expression when compared to the non-differentiated stage in C2C12 myoblasts. Expression showed a significant increase in dfd13 myoblasts when compared to the non-differentiated stage and also significant on days 4,7 , and 10 compared to C2C12 myoblasts (Fig 6D).

\section{Microtubule-associated light chain-3b expression is increased but autophagic flux is decreased during dystrophin-deficient myoblast differentiation}

Autophagy related genes were highly activated, and the next step was to determine whether a double-membrane vesicle, known as autophagosome, had been formed. The conversion of Light Chain-3B (LC3B-I) to LC3B-II can be considered to represent total autophagosome formation in myoblasts during differentiation.

As shown in figure 7A, expression of LC3B-I and LC3B-II is increased in both differentiating C2C12 and dfd13 myoblasts when compared to the respective non-differentiated myoblasts. LC3B-I expression in differentiated dfd13 myoblasts was significantly higher $(\mathrm{p}<0.01)$ at day 10 when compared to differentiated C2C12 myoblasts (Fig 7B). LC3B-II was found to accumulate upon differentiation in both types of myoblasts, but was higher in dfd13 myoblasts, with a significant difference on day 10 ( $p<0.05)$ when compared with to C2C12 myoblasts (Fig 7C). However, the LC3B-II/LC3B-I ratio showed a reduction in dfd13 myoblasts compared to $\mathrm{C} 2 \mathrm{C} 12$ myoblasts upon differentiation and was significantly different $(p<0.05)$ (Fig 7D).

LC3B-Il levels correlate with the number of autophagosomes formed; however, this could indicate either the up-regulation of autophagosome formation or a blockage in autophagic degradation. Besides, it does not conclusively indicate autophagic degradation. Therefore, further analysis was performed to determine autophagic flux, with a lysosome inhibitor used as a positive control.

To accurately determine autophagic activity, measuring the increase in the number of autophagosomes is required. Previous data showed that LC3B conversion was increased in $\mathrm{C} 2 \mathrm{C} 12$ but reduced in dfd13 myoblasts upon differentiation; however, the results from measuring LC3B conversion alone could be inappropriately interpreted. LC3B-II itself has been reported to be degraded by autophagy and also tends to be more sensitive than LC3B-I during immunoblotting analysis (30). Autophagosome formation is an intermediate stage of autophagy, and there could be either the generation of autophagosomes or the blocked conversion of autolysosomes. Therefore, an accurate measurement of autophagic flux is required. In this study a Cyto-ID autophagy detection kit was utilized which selectively labeled autophagic vacuoles independent of the LC3B protein, thus eliminating the need for transfection. Chloroquine was used as a control as it passively diffuses into lysosomes and increases the $\mathrm{pH}$, thus inhibiting lysosome function and blocking fusion with autophagosomes to become an autolysosome.

Page $8 / 27$ 
Figure 7 presents the density plots $(\mathrm{E}-\mathrm{H})$ and histograms (I-L) from flow cytometry-based profiling for both $\mathrm{C} 2 \mathrm{C} 12$ and dfd13 myoblasts in the non-differentiated and differentiated state. From the density plot images, the size and complexity of each myoblast can be determined. Based on the density plot images, it can be seen that the dfd13 myoblasts (Fig 7F) are smaller and less complex than $\mathrm{C} 2 \mathrm{C} 12$ myoblasts (Fig 7E), although the differences between these cells are not obvious. After differentiation, it can be seen that C2C12 cells become more complex (Fig 7G) while dfd13 cells remain the same (Fig 7H). A Cyto-ID autophagy detection kit and FITC were utilized to label/stain autophagosomes. The collected data were gated and analyses were performed based on the myoblasts in the gated region. Histograms were then plotted, as shown in Fig 7l-L; the black line indicates the unstained myoblasts (negative control), the blue line stained myoblasts, and the red line stained myoblasts treated with chloroquine (positive control). From the histograms, data were gated based on the intensity $\left(10^{1}-10^{3}\right)$ (X-axis) and then the percentage obtained.

Generally, both treated and non-treated myoblasts showed a reduction in autophagosome counts after 10 days of differentiation. Non-treated myoblasts had fewer autophagosomes when compared to treated myoblasts, which is due to chloroquine acting as a lysosome inhibitor, preventing autophagosome from fusing with lysosomes and causing the accumulation of autophagosomes (Figure I-L). As illustrated in Figure 7M, the number of autophagosomes was significantly decreased after 10 days of differentiation in both non-treated C2C12 myoblasts $(p<0.05)$ and dfd13 myoblasts $(p<0.05)$ when compared to the non-differentiated stage. It was also significantly decreased in treated C2C12 myoblasts $(p<0.005)$ and dfd13 myoblasts $(p<0.005)$ after 10 days of differentiation. There was a significant reduction in autophagosomes in dfd13 myoblasts when compared to $\mathrm{C} 2 \mathrm{C} 12$ myoblasts at the equivalent stages and treatment.

From these results, autophagic flux can be determined. Autophagic flux can be calculated by subtracting the chloroquinetreated from the untreated myoblasts, which enables the total number of non-fused autophagosomes to be measured and also the total number of autophagosomes formed. As depicted in Figure 7N, autophagic flux was increased in C2C12 myoblasts after 10 days of differentiation, whereas it was reduced in dfd13 myoblasts. When compared to non-differentiated $\mathrm{C} 2 \mathrm{C} 12$ myoblasts, the number of autophagosomes in dfd13 myoblasts was significantly higher $(p<0.05)$ and was slightly higher in differentiated dfd13 myoblasts compared to differentiated C2C12 myoblasts.

Overall, autophagy activity was decreased upon differentiation in dystrophin-deficient myoblasts. Based on the flow cytometry analysis, autophagic flux was decreased, as the total number of autophagosomes detected was higher in dfd13 myoblasts (both non-differentiated and differentiated) when compared to C2C12 myoblasts. Besides, autophagy was 2-fold higher in nondifferentiated myoblasts (proliferation state) compared to the differentiated state.

\section{Discussion}

\section{Dystrophin-deficient myoblasts do not achieve terminal differentiation}

In this experiment, it has been established that dystrophin-deficient myoblast differentiation is impaired when in a low mitogen medium for 10 days based on morphological analysis (Fig 1A and B) and the detection of F-MyHC expression (Fig 1E). Differentiation analyses showed that fewer myotubes were formed, and no/less F-MyHC expression was detected. However, pan-myosin was only expressed at the end of differentiation on day 10 in dfd13 myoblasts. Desmin expression levels were found to be higher at the end of the differentiation period. Desmin is a marker for newly formed fibers and is a class-III intermediate filament found in muscle cells that connect myofibrils to each other, as well as to the plasma membrane of myofibres. An in vivo study by Smythe et al. (2001) indicated that desmin expression is up-regulated during myogenesis and demonstrated that desmin(-/-) showed delays in myotube regeneration 5 days after transplantation when compared to the control where desmin was detected (31). Desmin also has been reported overexpressed at the molecular level in DMD patients $(32,33)$. In this study, it can be suggested that the integrative element in myofibres was generated/developed earlier than myotube formation in the differentiating dystrophin-deficient myoblasts, although full/terminal differentiation was not achieved. 
During myogenesis, proliferating myoblasts withdraw from the cell cycle and differentiate into myotubes. Cyclin-dependent Kinase (CDK) inhibitor p21 and retinoblastoma protein $(\mathrm{Rb})$ have been shown to play a critical role in establishing the postmitotic state by permitting the transcription of the S-phase promoting gene during myogenesis (34). Most mitogens have been shown to promote myoblast proliferation. In contrast, insulin-like growth factors (Igf-1 and Igf-2) trigger myoblast differentiation. It is unknown how Igf-2 is controlled during the initiation of differentiation. A previous study showed that Igf-2 up-regulates its own gene expression via the Akt/PKB pathway (35). Elevation of Igf-2 mRNA was found in C2 myoblasts after 24 hours' culture in differentiation medium containing 2-10\% horse serum (36), while Erbay et al. (2003) suggested that mTOR regulates Igf-2 production at the transcriptional level when cultured under low mitogen conditions $(37,38)$. There has also been a study suggesting that myoblasts under low mitogen conditions have elevated levels of IGFBP5, which helps concentrate IGF2 to a threshold level which triggers the IGF-1R pathway leading to differentiation $(39,40)$. However, in this study levels of Igf$1 / 2$ could not be examined in the culture system utilized, i.e. Igf- $1 / 2$ secreted/present in the media. Therefore, further study needs to be undertaken to examine this aspect.

\section{Elevation of PTEN affects PI3K/AKT regulation in dystrophin-deficient myoblasts}

The PI3K/Akt pathway is a highly conserved pathway for the regulation of skeletal muscle growth and is activated by the binding of Igf-1 to its receptor, Igf1-r. This binding leads to intrinsic tyrosine kinase activation, as well as auto-phosphorylation, thus activating PI3K/Akt signaling for myoblast differentiation via a series of phosphorylation events. This signaling is negatively regulated by PTEN. In this study PTEN was shown to be elevated in dfd13 myoblasts, which affected PI3K activation, as it was found to be decreased during differentiation (Fig 2D), indicating that PI3K regulation is altered in dfd13 myoblasts. PTEN acts as lipid phosphatase and plays a role in removing the phosphate group present on the inositol ring of $\mathrm{PIP}_{3}$ to produce $\mathrm{PIP}_{2}$. In contrast, $\mathrm{PI} 3 \mathrm{~K}$ reverses this event by phosphorylating $\mathrm{PIP}_{2}$ to become $\mathrm{PIP}_{3}$.

PI3K exists as a heterodimer consisting of two subunits; regulatory and catalytic. When cells are stimulated, p85 binds to tyrosine-phosphorylated IRS, and phosphorylated-p85 changes the conformation of p110 and thus mediates the p110 subunit to translocate to the membrane and increases its enzymatic activity such as $\mathrm{PIP}_{2}$ phosphorylation. $\mathrm{PIP}_{3}$ helps to recruit Akt by binding the plekstrin homolog $(\mathrm{PH})$ domain at the $\mathrm{N}$-terminal of Akt to the cell membrane. PTEN in turn acts as a negative regulator by dephosphorylating $\mathrm{PIP}_{3}$ to form $\mathrm{PIP}_{2}$, and thereby reduces the available docking sites for Akt to bind to before activation. PTEN is mainly found in the cytosol and nucleus. The $\mathrm{N}$-terminal possesses a $\mathrm{PIP}_{2}$-binding motif while the $\mathrm{C}$ terminal contains a serine/threonine phosphorylation, Ser380, which regulates its stability and activity, as well as membrane recruitment. The main $\mathrm{C} 2$ domain contains basic residues that are essential for membrane binding. Phosphorylation of PTEN is considered to result in a closed conformation which is the inactive form. In this conformation, it has been proposed that the phosphorylated C-terminal interacts with the positively charged C2 domain and remains in the cytoplasm (41). As the membrane integrity is disrupted due to the absence of dystrophin, it can be suggested that cytoplasmic-membrane activity is altered in dystrophin-deficient myoblasts and thus there is impaired PTEN-PI3K regulation. In this study, phosphorylation of PI3K-p85 (regulatory subunit) was examined to observe PI3K activation. When cells are stimulated by the receptor, intrinsic tyrosine kinase phosphorylates p85 at Tyr458, causing a conformational change to p110 (the catalytic subunit) which increases its enzymatic activity for PIP 2 . From this data, it seems that high PTEN expression decreased PI3K activity, thus affecting its downstream protein activation.

Feron and colleagues (2009) reported that increased PTEN expression caused the deregulation of the PI3K/Akt pathway in dystrophin-deficient muscle present in GRMD dogs. This was also observed in muscle sections from 3- to 36-month old animals and indicates that the PI3K/Akt pathway is a long-term alteration. A more recent study by Alexander et al. (2014) proposed that overexpression of micro-RNA-486 improved muscle physiology and performance in dystrophin-deficient mice (42). Micro-RNA-486 is a muscle-enriched micro-RNA that is markedly reduced in the muscle of dystrophin-deficient mice and DMD patient muscles $(3,43)$. 


\section{Inactivation of rictor-mTOR2 impaired Akt phosphorylation in dystrophin-deficient myoblasts}

In this study, it was found that Akt is not/less activated in dfd13 myoblasts, as phosphorylation at Ser473 or Thr308 was not detected. However, in C2C12 myoblasts Akt was phosphorylated at Ser473, and the activated form accumulated upon differentiation. Immunofluorescence analysis showed that phosphorylated-Akt (Ser473) is localized to the membrane of C2C12 myotubes but found less often in dfd13 myoblasts. During biosynthesis, nascent Akt is phosphorylated at Thr450 within the turn motif site and localized to the cytosol when in its inactive conformation. In the presence of signals via PI3K activation, Akt is recruited to the membrane through the binding of plekstrin homolog $(\mathrm{PH})$ to $\mathrm{PIP}_{3}$. Once bound, the conformation is changed and this event unmasks two residues for phosphorylation, Ser473, and Thr308. In this study, fewer Akt-phosphorylated at Ser473 was detected in dystrophin-deficient myoblasts, indicating that Akt is inactivated/less activated. According to Sarbassov et al. (2005), PDK1 has a better target on Akt phosphorylated at Ser473 than non-phosphorylated Akt (26). In line with the results shown in Figure 3, there is no Thr308 phosphorylation if Ser473 phosphorylation does not occur. However, the expression of total Akt did not show any significant difference when compared between both types of myoblast.

Akt is a downstream protein of PI3K. It plays an important role and is recognized as one of the most critical pathways in the regulation of cell viability and maintenance in skeletal muscle mass (44). Therefore, its expression pattern in dystrophindeficient myoblasts was investigated, as previously dfd13 myoblasts have been reported to undergo apoptosis when cultured in DM (45). As expected, the immunoblot data showed that Akt was not found/less activated in differentiating dfd13 myoblasts. Surprisingly, it was found that Akt was only activated by phosphorylation at Ser473 in C2C12 myoblasts. Akt was not phosphorylated at Thr308 in either type of myoblasts and consequently mouse embryonic fibroblast (MEF) cells were used as a positive control (gift from Dr. Adil Rashid, University of Birmingham) for this activation site.

Since Akt was found to only be phosphorylated at Ser473 the protein responsible for this phosphorylation event was investigated. It is known that mTORC2, specifically, rictor, can directly phosphorylate Akt at Ser473 in vitro, and this facilitated Thr308 phosphorylation by PDK1 in drosophila (26). In this study, it was found that rictor is inactivated in both undifferentiated and differentiated dfd13 cells, which explains the absence of phosphorylated-Akt (Ser473) observed; however, the regulator for rictor activation in myoblasts remains unknown. Rictor phosphorylation has been shown to require mTORC1-activated p70S6K in MEF (46), HEK293 (47), and HeLa cells (48). Recently, it has been shown that PTEN negatively regulates mTORC2 signaling in glioma (49). As PTEN was found to be highly expressed in dfd13 myoblasts, inactivation of rictor can be seen as a consequence of this event and presumably represents the same scenario as in myoblasts. From these results, it can also be suggested that inactivation of Akt is thought to affect other kinases, such as protein kinase C (PKC), and focal adhesion proteins, such as integrin-linked-kinase (ILK). Alterations to these kinases have been reported as one of the factors that cause MD.

\section{Excessive formation of autophagosome in dystrophin-deficient myoblast}

In skeletal muscle, autophagy is transiently activated and continues for only a few days $(11,50)$; it is regulated via FoxO3. Generally, it can be seen that autophagosomes are formed in both types of myoblast during differentiation, as the autophagyrelated protein involved in the initiation and elongation process was found to be increased. The ratio of LC3B-I converted to LC3B-II also reflected the formation of autophagosomes during differentiation (Fig 7D).

In this study, it can be seen that autophagosome formation is modulated via FoxO3 in dfd13 myoblasts. Inactivation of Akt allowed the active-form of FoxO3 (unphosphorylated) to translocate into the nucleus and trigger the expression of autophagyrelated genes. FoxO3 is required for the transcriptional regulation of LC3B and also for transcriptional regulation of MAFbx and MuRF1. This transcriptional regulation leads to protein degradation via the autophagy-lysosome pathway and the ubiquitinproteosome pathway, respectively. However, autophagy in C2C12 myoblasts seems to be only partially FoxO3-mediated, as

Page $11 / 27$ 
subsequent activation of Akt inhibited FoxO3 and suppressed its translocation to the nucleus and the targeting of autophagyrelated gene activation. Activated-Akt, through phosphorylation at Ser473 by rictor-mTORC2, also contributes to autophagy activation during C2C12 myoblast differentiation $(27,51)$.

\section{Modulation of Atg5-dependent autophagy during dystrophin-deficient myoblasts differentiation}

Autophagy is responsible for removing unfolded proteins as well as dysfunctioning organelles and has been reported to be constantly active within skeletal muscle. Recently, increased autophagy has been reported to protect differentiating myoblasts from apoptotic cell death (52). Several autophagy-related genes are known to be involved in the formation of autophagosomes, and FoxO3 has been shown to induce multiple autophagy-related genes, including LC3B transcription in skeletal muscle $(29,53)$. LC3B is an isoform of LC3 which plays a critical role in autophagy via post-translational modification.

LC3B is a subunit of microtubule-associated protein 1 (MAP1LC3B), and LC3 is cleaved by Atg4 to become cytosolic LC3B-I. LC3B-I is then converted to lipidated-LC3B-II through the conjugation of membrane lipid phosphatidylethanolamine (PE), which involves the E1-like enzyme ubiquitin, Atg7, and the E2-like enzyme, Atg10. LC3B-Il then binds to the isolation membrane and mediates membrane elongation until the edges fuse to form an autophagosome. The isolation membrane appears when cells are placed under starvation conditions. Atg7 also conjugate Atg5 to Atg12 to form the Atg5-Atg12 complex and then bind to the isolation membrane with Atg16. This complex binding is necessary for autophagosome formation.

In this study, the results showed that LC3B-I was increased in both types of myoblast due to the up-regulation of Beclin1 by nuclear-Fox03, which is increased in dystrophin-deficient myoblasts. Beclin1 forms a complex with Vsp34 and becomes a core component during the pre-autophagosome stage. This complex then binds to the ULK1/Atg13/FIP200/Atg101 complex before entering the elongation stage, when Atg7 catalyzes the ligation of Atg5 to Atg12 to become the Atg5-Atg12 complex. With the aid of the Atg5-Atg12 complex, Atg7 catalyzes the transfer of PE to LC3B-I, converting it to LC3B-II, resulting in the accumulation of LC3B-II within dfd13 myoblasts (Fig 7C). Therefore, autophagosomes can be formed even when Atg5 is not present and so it can be suggested that Atg 5 plays a booster role for autophagosome formation in dfd13 myoblasts.

Autophagy in C2C12 myoblasts might also be activated via PERK-mediated CHOP (54). Although the expression of both LC3B-I and LC3B-II in differentiating dfd13 myoblasts accumulates until day 10, the conversion ratio of LC3B-I to LC3B-II was reduced in dystrophin-deficient myoblasts, indicating that autophagy regulation is impaired.

It has been reported that autophagy is induced upon myoblast differentiation to eliminate pre-existing structures and proteins. This elimination occurs concomitantly with the myoblast fusion process before the formation of multinucleated myotubes. Based on the results presented, autophagy is activated in both types of myoblasts; however, overexpression of Atg7 and Atg5 in dystrophin-deficient myoblasts suggests that the ubiquitin-like system is impaired. This could affect cascade activation which in turn will affect autophagosome formation, as well as an autophagic flux in dystrophin-deficient myoblasts.

\section{Reduction in autophagic flux proves defective autophagy in dystrophin- deficient myoblasts}

Autophagy is responsible for the removal of unfolded protein as well as dysfunctioning organelles and has been reported to be constantly active within skeletal muscle (11). Beclin1 is the protein responsible for the initiation of autophagosome formation and is also known as phagophore, while Atg5 and Atg7 are ubiquitin-like enzymes involved in the autophagosome elongation process. In this study, Beclin1, Atg5, and Atg7 showed enhanced expression in dfd13 myoblasts. However, in C2C12 myoblasts there was only a slight increase in the expression of Beclin1 and Atg7 which persisted throughout the differentiation period. Consequently, autophagosome formation (maturation) and autophagic flux during myoblast differentiation were investigated via immunoblotting and flow cytometry analysis, respectively. 
From the data obtained the increased expression of autophagy-related genes (Atg5, Atg7 \& Beclin1) demonstrates excessive autophagosome formation in dfd13 myoblasts during differentiation; however, the conversion ratio of LC3B-I to LC3B-II was reduced in dystrophin-deficient myoblasts. Furthermore, although activation was higher, autophagic flux analysis showed a reduction upon differentiation and revealed that autophagy activity is decreased upon differentiation in dfd13 myoblasts (Fig

8). Therefore, it was suggested that after excessive autophagosome formation in non-differentiated dfd13 myoblasts could potentially undergo apoptosis during differentiation. Previously, our group has shown that the ratio of cleaved caspase-3 to uncleaved caspase-3 is increased in a derivative of dfd13 myoblasts, PD50A when cultured in DM (45).

DMD is mostly characterized by $\sim 2$ years of age and it progresses until the early 20 s. Therefore, the applicability of this finding to the understating of DMD patients may fit with the actual scenario. During the early stage of life (newborn to below 2 years), deficient myoblasts survive as most cells are actively proliferating and high levels of autophagy activation can prevent apoptosis. As age increases (>2 years old), most of the deficient myoblasts have been triggered for differentiation as the body develops and muscle size and functionality increase, but now autophagy starts to decrease and apoptosis begins. This is the most common period for when DMD is diagnosed. At the later stage ( 10 years-old), there is a progressive disruption to the muscle due to loss/damage, and support (wheelchair) is needed for mobility and undertaking daily life tasks. This state will worsen and patients will eventually die, commonly as a complication of respiratory muscle damage and cardiomyopathy.

\section{Conclusion}

PTEN-PI3K/Akt and its downstream proteins are perturbed in dystrophin-deficient myoblasts. From the data obtained, dystrophin-deficient myoblasts exhibit the high expression of autophagy-related proteins. However, a reduction in autophagy activity, as well as autophagy flux upon differentiation, indicates that autophagy is defective. This finding suggests a new mechanism for the reduction of autophagy in dystrophin-deficient myoblasts. All in all, we have come out with a schematic of autophagy modulation in dystrophin-deficient myoblasts (Figure 8). We showed that perturbation of the PTEN-PI3K/Akt pathway initially triggers excessive autophagosome formation, and subsequently there is a reduction in autophagic flux within dystrophin-deficient myoblasts.

\section{List Of Abbreviations}




\begin{tabular}{|c|c|}
\hline Atg5 & Autophagy related 5 \\
\hline Atg7 & Autophagy related 7 \\
\hline Atg10 & Autophagy related 10 \\
\hline Atg12 & Autophagy related 12 \\
\hline Atg13 & Autophagy related 13 \\
\hline Atg16 & Autophagy related 16 \\
\hline Atg101 & Autophagy related 101 \\
\hline BSA & Bovine Serum Albumin \\
\hline CDK & Cyclin-dependent Kinase \\
\hline $\mathrm{CHOP}$ & C/EBP homologous protein \\
\hline DAPI & 4',6-diamidino-2-phenylindole \\
\hline $\mathrm{CO}_{2}$ & Carbon dioxide \\
\hline DM & Differentiation Medium \\
\hline DMD & Duchenne Muscular Dystrophy \\
\hline DMEM & Dulbecco's Modified Eagle's medium \\
\hline DNA & Deoxyribonucleic Acid \\
\hline EDTA & Ethylenediaminetetra Acetic Acid \\
\hline ERK1/2 & extracellular-signal-regulated kinase-1/2 \\
\hline FIP200 & focal adhesion kinase family interacting protein of $200 \mathrm{kD}$ \\
\hline FBS & Fetal Bovine Serum \\
\hline FITC & Fluorescein Isothiocyanate \\
\hline MyHC & Myosin Heavy Chain \\
\hline F-MyHC & Fast Myosin Heavy Chain \\
\hline FoxO3 & Forkhead box 03 \\
\hline GM & Growth Medium \\
\hline GSK3 $\beta$ & Glycogen synthase kinase 3 beta \\
\hline GRMD & Golden Retriever Muscular Dystrophy \\
\hline HEK293 & human embryonic kidney-293 \\
\hline IGF & Insulin-Like Growth Factor \\
\hline IGF-1 & Type 1 lgf \\
\hline IGF-2 & Type 2 lgf \\
\hline IGF-1R & Type 1 Igf Receptor \\
\hline IGF-2R & Type 2 Igf Receptor \\
\hline ILK & integrin-linked-kinase \\
\hline IRS & Insulin Receptor Substrate \\
\hline
\end{tabular}

Page $14 / 27$ 


\begin{tabular}{|c|c|}
\hline LC3B & light chain 3B \\
\hline MAP1LC3B & microtubule-associated protein 1 light chain 3B \\
\hline MEF & mouse embryonic fibroblast \\
\hline mTOR & mammalian target of rapamycin \\
\hline mTORC1 & mammalian target of rapamycin complex 1 \\
\hline mTORC2 & rapamycin-insensitive protein complex 2 \\
\hline MD & Muscular Dystrophy \\
\hline $\mathrm{MyHC}$ & Myosin Heavy Chain \\
\hline $\mathrm{NaCl}$ & sodium chloride \\
\hline NP-40 & nonyl-phenoxypolyethoxylethanol \\
\hline PAGE & Polyacrylamide Gel Electrophoresis \\
\hline PBS & Phosphate-Buffered Saline \\
\hline PE & phosphatidylethanolamine \\
\hline $\mathrm{PH}$ & plekstrin homolog \\
\hline PDK1 & Phosphoinositide-dependent kinase-1 \\
\hline $\mathrm{PIP}_{3}$ & Phosphatidylinositol $(3,4,5)$-trisphosphate \\
\hline $\mathrm{PIP}_{2}$ & Phosphatidylinositol (4,5)-trisphosphate \\
\hline PFA & Paraformaldehyde \\
\hline $\mathrm{PI} 3 \mathrm{~K}$ & Phosphoinositide 3-kinases \\
\hline PTEN & Phosphatase and tensin homolog \\
\hline p70s6K & Ribosomal protein S6 kinase beta-1 \\
\hline mRNA & Messenger ribonucleic acid rpm revolutions per \\
\hline $\mathrm{Rb}$ & retinoblastoma \\
\hline $\mathrm{rpm}$ & Revolutions Per Minute \\
\hline SDS & sodium dodecyl sulfate \\
\hline ULK1 & Unc-51 like autophagy activating kinase \\
\hline WCL & Whole cell lysate \\
\hline
\end{tabular}

\section{Declarations}

\section{Ethics approval and consent to participate}

Not applicable

\section{Consent for publication}

All authors read and approved the paper 


\section{Availability of data and material}

The datasets used and/or analyzed during the study are available from the corresponding author

\section{Competing Interests}

The authors report no conflicts of interest. The authors alone are responsible for the content and writing of the paper.

\section{Funding}

This research was performed with the financial support of Universiti Kebangsaan Malaysia (GUP-2019-030) \& Majlis Amanah Rakyat (MARA).

\section{Authors' Contribution}

Design the experiments: MDY CH-C. Performed the experiments: MDY. Analyzed the data: MDY. Wrote the paper: MDY $\mathrm{CH}-\mathrm{C}$. All authors read and approved the final manuscript.

\section{Acknowledgments}

The authors would like to thank Dr. Janet Smith and Dr. Neil Hotchin for their guidance and advice during research work. Also thank Dr. Melissa Grant (School of Dentistry, University of Birmingham), Dr. Debbie Cunningham and Dr. Trushar Patel (School of Biosciences, University of Birmingham) Dr. Zubair Ahmed (Medical School, University of Birmingham), Dr. Shabana Begum and Dr. Adil Rashid (University of Birmingham) for reagents supplement, Dr. Matthew MacKenzie (Institute for Biomedical Sciences (IBR), University of Birmingham) for flow cytometry data acquisition, Professor John K. Heath (University of Birmingham) and Professor Theodore Fotsis (Institute of Molecular Biology \& Biotechnology, University of loannina, Greece) for helpful comments and idea throughout the work.

\section{References}

1. Hoffman EP, Brown RH, Kunkel LM. Dystrophin: The protein product of the duchenne muscular dystrophy locus. Cell [Internet]. 1987 Dec 24 [cited 2017 Feb 4];51(6):919-28. Available from:

http://www.cell.com/article/0092867487905794/fulltext

2. Meryon E. On Granular Degeneration of the Voluntary Muscles. Med Chir Trans. 1866;35:73-84.1.

3. Vieira NM, Spinazzola JM, Alexander MS, Moreira YB, Kawahara G, Gibbs DE, et al. Repression of phosphatidylinositol transfer protein a ameliorates the pathology of Duchenne muscular dystrophy. Proc Natl Acad Sci [Internet]. 2017;114(23):6080-5. Available from: http://www.pnas.org/lookup/doi/10.1073/pnas.1703556114

4. Chamberlain JS. Duchenne muscular dystrophy models show their age. Cell. 2010;143(7):1040-2.

5. Sumitani S, Goya K, Testa JR, Kouhara H, Kasayama S. Akt1 and Akt2 differently regulate muscle creatine kinase and myogenin gene transcription in insulin-induced differentiation of C2C12 myoblasts. Endocrinology. 2002;143(3):820-8.

6. Arun RP, Sivanesan Di, Vidyasekar P, Verma RS. PTEN/FOXO3/AKT pathway regulates cell death and mediates morphogenetic differentiation of Colorectal Cancer Cells under Simulated Microgravity. Sci Rep. 2017;7(1).

7. Qin Y, Huo Z, Song X, Chen X, Tian X, Wang X. mir-106a regulates cell proliferation and apoptosis of colon cancer cells through targeting the PTEN/PI3K/AKT signaling pathway. Oncol Lett [Internet]. 2017; Available from: http://www.spandidos-publications.com/10.3892/ol.2017.7715 
8. Dogra C, Changotra H, Wergedal JE, Kumar A. Regulation of phosphatidylinositol 3-kinase (PI3K)/Akt and nuclear factorkappa B signaling pathways in dystrophin-deficient skeletal muscle in response to mechanical stretch. J Cell Physiol [Internet]. 2006 Sep [cited 2017 Feb 4];208(3):575-85. Available from: http://www.ncbi.nlm.nih.gov/pubmed/16741926

9. Haslett JN, Sanoudou D, Kho AT, Han M, Bennett RR, Kohane IS, et al. Gene expression profiling of Duchenne muscular dystrophy skeletal muscle. Neurogenetics [Internet]. 2003;4(4):163-71. Available from: http://dx.doi.org/10.1007/s10048003-0148-x

10. Feron M, Guevel L, Rouger K, Dubreil L, Arnaud M-C, Ledevin M, et al. PTEN Contributes to Profound PI3K/Akt Signaling Pathway Deregulation in Dystrophin-Deficient Dog Muscle. Am J Pathol [Internet]. 2017 Feb 4;174(4):1459-70. Available from: http://dx.doi.org/10.2353/ajpath.2009.080460

11. De Palma C, Perrotta C, Pellegrino P, Clementi E, Cervia D. Skeletal muscle homeostasis in Duchenne muscular dystrophy: Modulating autophagy as a promising therapeutic strategy. Front Aging Neurosci. 2014;6(JUL).

12. Peter AK, Crosbie RH. Hypertrophic response of Duchenne and limb-girdle muscular dystrophies is associated with activation of Akt pathway. Exp Cell Res. 2006;312(13):2580-91.

13. Boppart MD, Burkin DJ, Kaufman SJ. Activation of AKT signaling promotes cell growth and survival in a7 $\beta 1$ integrinmediated alleviation of muscular dystrophy. Biochim Biophys Acta [Internet]. 2011 Apr 7;1812(4):439-46. Available from: http://www.ncbi.nlm.nih.gov/pmc/articles/PMC3046458/

14. Glick D, Barth S, Macleod KF. AutophagGlick, D., Barth, S., \& Macleod, K. F. (2010). Autophagy: cellular and molecular mechanisms. Journal of Pathology The, 221, 3-12. http://doi.org/10.1002/path.2697.Autophagyy: cellular and molecular mechanisms. J Pathol [Internet]. 2010;221:3-12. Available from: http://www.ncbi.nlm.nih.gov/pubmed/20225336

15. Grumati P, Bonaldo P. Autophagy in skeletal muscle homeostasis and in muscular dystrophies. Cells [Internet]. 2012 Jan 26 [cited 2016 Sep 7];1(3):325-45. Available from: http://www.mdpi.com/2073-4409/1/3/325/htm

16. Tchevkina E, Komelkov A. Protein Phosphorylation as a Key Mechanism of mTORC1/2 Signaling Pathways Y1 - 2012-0906 N1 38801 UR http://www.intechopen.com/books/export/citation/Zotero/protein-phosphorylation-in-humanhealth/protein-phosphorylation-as-a-key-mechanism-of-mtorc1-2-si. In: Protein Phosphorylation in Human Health.

17. Masiero E, Agatea L, Mammucari C, Blaauw B, Loro E, Komatsu M, et al. Autophagy Is Required to Maintain Muscle Mass. Cell Metab. 2009;10(6):507-15.

18. Neel BA, Lin Y, Pessin JE. Skeletal Muscle Autophagy: A New Metabolic Regulator. Trends Endocrinol Metab [Internet]. 2013 Dec 29;24(12):10.1016/j.tem.2013.09.004. Available from: http://www.ncbi.nlm.nih.gov/pmc/articles/PMC3849822/

19. De Palma C, Morisi F, Cheli S, Pambianco S, Cappello V, Vezzoli M, et al. Autophagy as a new therapeutic target in Duchenne muscular dystrophy. Cell Death Dis [Internet]. 2012;3(11):e418. Available from:

http://www.pubmedcentral.nih.gov/articlerender.fcgi?

artid=3542595\&tool=pmcentrez\&rendertype=abstract\%5Cnhttp://www.nature.com/doifinder/10.1038/cddis.2012.159

20. Yaffe D, Saxel O. Serial passaging and differentiation of myogenic cells isolated from dystrophic mouse muscle. Nature. 1977;

21. Smith J, Schofield PN. The effects of fibroblast growth factors in long-term primary culture of dystrophic (mdx) mouse muscle myoblasts. Exp Cell Res. 1994;210(1):86-93.

22. Smith J, Schofield PN. Stable integration of an mdx skeletal muscle cell line into dystrophic (mdx) skeletal muscle: evidence for stem cell status. Cell Growth Differ [Internet]. 1997;8(8):927-34. Available from:

http://www.ncbi.nlm.nih.gov/entrez/query.fcgi?cmd=Retrieve\&db=PubMed\&dopt=Citation\&list_uids=9269902

23. Suzuki K, Bose P, Leong-Quong RYY, Fujita DJ, Riabowol K. REAP: A two minute cell fractionation method. BMC Res Notes [Internet]. 2010 Nov 10;3:294. Available from: http://www.ncbi.nlm.nih.gov/pmc/articles/PMC2993727/

24. Schiaffino S, Reggiani C. Fiber types in mammalian skeletal muscles. Physiol Rev [Internet]. 2011;91(4):1447-531. Available from: http://www.ncbi.nlm.nih.gov/pubmed/22013216

25. Wan X, Helman LJ. Levels of PTEN protein modulate Akt phosphorylation on serine 473, but not on threonine 308, in IGF-IIoverexpressing rhabdomyosarcomas cells. Oncogene [Internet]. 2003;22(50):8205-11. Available from: 
http://dx.doi.org/10.1038/sj.onc.1206878

26. Sarbassov DD, Guertin DA, Ali SM, Sabatini DM. Phosphorylation and Regulation of Akt/PKB by the Rictor-mTOR Complex. Science (80-) [Internet]. 2005 Feb 17;307(5712):1098 LP - 1101. Available from:

http://science.sciencemag.org/content/307/5712/1098.abstract

27. Shu L, Houghton PJ. The mTORC2 complex regulates terminal differentiation of C2C12 myoblasts. Mol Cell Biol [Internet]. 2009;29(17):4691-700. Available from: http://www.pubmedcentral.nih.gov/articlerender.fcgi? artid $=2725723 \&$ tool=pmcentrez\&rendertype=abstract

28. Rosner M, Siegel N, Valli A, Fuchs C, Hengstschläger M. MTOR phosphorylated at S2448 binds to raptor and rictor. Amino Acids. 2010;38(1):223-8.

29. Mammucari C, Milan G, Romanello V, Masiero E, Rudolf R, Del Piccolo P, et al. FoxO3 Controls Autophagy in Skeletal Muscle In Vivo. Cell Metab. 2007;6(6):458-71.

30. Mizushima N, Yoshimori T. How to interpret LC3 immunoblotting. Vol. 3, Autophagy. 2007. p. 542-5.

31. Smythe GM, Davies MJ, Paulin D, Grounds MD. Absence of desmin slightly prolongs myoblast proliferation and delays fusion in vivo in regenerating grafts of skeletal muscle. Cell Tissue Res [Internet]. 2001;304(2):287-94. Available from: http://dx.doi.org/10.1007/s004410100366

32. Haslett JN, Sanoudou D, Kho AT, Bennett RR, Greenberg SA, Kohane IS, et al. Gene expression comparison of biopsies from Duchenne muscular dystrophy (DMD) and normal skeletal muscle. Proc Natl Acad Sci [Internet]. 2002 Nov 12;99(23):15000-5. Available from: http://www.pnas.org/content/99/23/15000.abstract

33. Tsoupri E, Capetanaki Y. Myospryn: A multifunctional desmin-associated protein. Histochemistry and Cell Biology. 2013.

34. Blagosklonny M V., Pardee AB. The restriction point of the cell cycle. Vol. 1, Cell cycle (Georgetown, Tex.). 2002. p. 103-10.

35. Duan C, Liimatta MB, Bottum OL. Insulin-like Growth Factor (IGF)-I Regulates IGF-binding Protein-5 Gene Expression through the Phosphatidylinositol 3-Kinase, Protein Kinase B/Akt, and p70 S6 Kinase Signaling Pathway. J Biol Chem [Internet]. 1999 Dec 24 [cited 2017 Feb 4];274(52):37147-53. Available from:

http://www.jbc.org/cgi/content/short/274/52/37147

36. Tollefsen SE, Lajara R, McCusker RH, Clemmons DR, Rotwein P. Insulin-like growth factors (IGF) in muscle development. Expression of IGF-I, the IGF-I receptor, and an IGF binding protein during myoblast differentiation. J Biol Chem. 1989;264(23):13810-7.

37. Erbay E, Park IH, Nuzzi PD, Schoenherr CJ, Chen J. IGF-II transcription in skeletal myogenesis is controlled by mTOR and nutrients. J Cell Biol. 2003;163(5):931-6.

38. Torrente Y, Bella P, Tripodi L, Villa C, Farini A. Role of Insulin-Like Growth Factor Receptor 2 across Muscle Homeostasis: Implications for Treating Muscular Dystrophy. Cells. 2020;

39. James PL, Jones SB, Busby WH, Clemmons DR, Rotwein P. A highly conserved insulin-like growth factor-binding protein (IGFBP-5) is expressed during myoblast differentiation. J Biol Chem [Internet]. 1993;268(30):22305-12. Available from: http://www.ncbi.nlm.nih.gov/pubmed/7693664

40. Florini JR, Ewton DZ, Coolican SA. Growth hormone and the insulin-like growth factor system in myogenesis. Endocr Rev. 1996;17(5):481-517.

41. Vazquez F, Matsuoka S, Sellers WR, Yanagida T, Ueda M, Devreotes PN. Tumor suppressor PTEN acts through dynamic interaction with the plasma membrane. Proc Natl Acad Sci U S A [Internet]. 2006 Mar 7 [cited 2017 Feb 4];103(10):3633-8. Available from: http://www.pnas.org/cgi/content/short/103/10/3633

42. Alexander MS, Casar JC, Motohashi N, Vieira NM, Eisenberg I, Marshall JL, et al. MicroRNA-486 - dependent modulation of DOCK3 / PTEN / AKT signaling pathways improves muscular dystrophy - associated symptoms. J Clin Invest. 2014;124(6):2651-67.

43. Eisenberg I, Eran A, Nishino I, Moggio M, Lamperti C, Amato AA, et al. Distinctive patterns of microRNA expression in primary muscular disorders. Proc Natl Acad Sci U S A [Internet]. 2007 Oct 23 [cited 2017 Feb 4];104(43):17016-21. Available from: http://www.pnas.org/cgi/content/short/104/43/17016

Page $18 / 27$ 
44. Egerman MA, Glass DJ. Signaling pathways controlling skeletal muscle mass. Crit Rev Biochem Mol Biol [Internet]. 2014 Jan 18;49(1):59-68. Available from: http://www.ncbi.nlm.nih.gov/pmc/articles/PMC3913083/

45. Hung-chih C. Function of caveolin-1 and caveolin-3 i muscular dystrophy. University of Birmingham; 2013.

46. Martin TD, Dennis MD, Gordon BS, Kimball SR, Jefferson LS. mTORC1 and JNK coordinate phosphorylation of the p70S6K1 autoinhibitory domain in skeletal muscle following functional overloading. Am J Physiol - Endocrinol Metab. 2014;

47. Gleason CE, Oses-Prieto JA, Li KH, Saha B, Situ G, Burlingame AL, et al. Phosphorylation at distinct subcellular locations underlies specificity in mTORC2-mediated activation of SGK1 and Akt. J Cell Sci. 2019;

48. Julien LA, Carriere A, Moreau J, Roux PP. mTORC1-activated S6K1 phosphorylates Rictor on threonine 1135 and regulates mTORC2 signaling. Mol Cell Biol [Internet]. 2010;30(4):908-21. Available from:

http://www.ncbi.nIm.nih.gov/pubmed/19995915\%5Cnhttp://www.ncbi.nlm.nih.gov/pmc/articles/PMC2815569/pdf/060109.pdf

49. Bhattacharya K, Maiti S, Mandal C. PTEN negatively regulates mTORC2 formation and signaling in grade IV glioma via Rictor hyperphosphorylation at Thr1135 and direct the mode of action of an mTORC1/2 inhibitor [Internet]. Vol. 5, Oncogenesis. Macmillan Publishers Limited; 2016. p. e227. Available from: http://dx.doi.org/10.1038/oncsis.2016.34

50. Mizushima N. Methods for monitoring autophagy. Vol. 36, International Journal of Biochemistry and Cell Biology. 2004. p. 2491-502.

51. Ge Y, Chen J. Mammalian target of rapamycin (mTOR) signaling network in skeletal myogenesis. J Biol Chem [Internet]. 2012 Dec 21 [cited 2017 Feb 4];287(52):43928-35. Available from: http://www.jbc.org/cgi/content/short/287/52/43928

52. McMillan EM, Quadrilatero J. Autophagy is required and protects against apoptosis during myoblast differentiation. Biochem J [Internet]. 2014 Aug 7;462(2):267 LP - 277. Available from:

http://www.biochemj.org/content/462/2/267.abstract

53. Milan G, Romanello V, Pescatore F, Armani A, Paik JH, Frasson L, et al. Regulation of autophagy and the ubiquitinproteasome system by the FoxO transcriptional network during muscle atrophy. Nat Commun. 2015;

54. Matsumoto H, Miyazaki S, Matsuyama S, Takeda M, Kawano M, Nakagawa H, et al. Selection of autophagy or apoptosis in cells exposed to ER-stress depends on ATF4 expression pattern with or without CHOP expression. Biol Open [Internet]. 2013;2(10):1084-90. Available from: http://www.pubmedcentral.nih.gov/articlerender.fcgi? artid $=3798192 \&$ tool=pmcentrez\&rendertype $=$ abstract

\section{Figures}



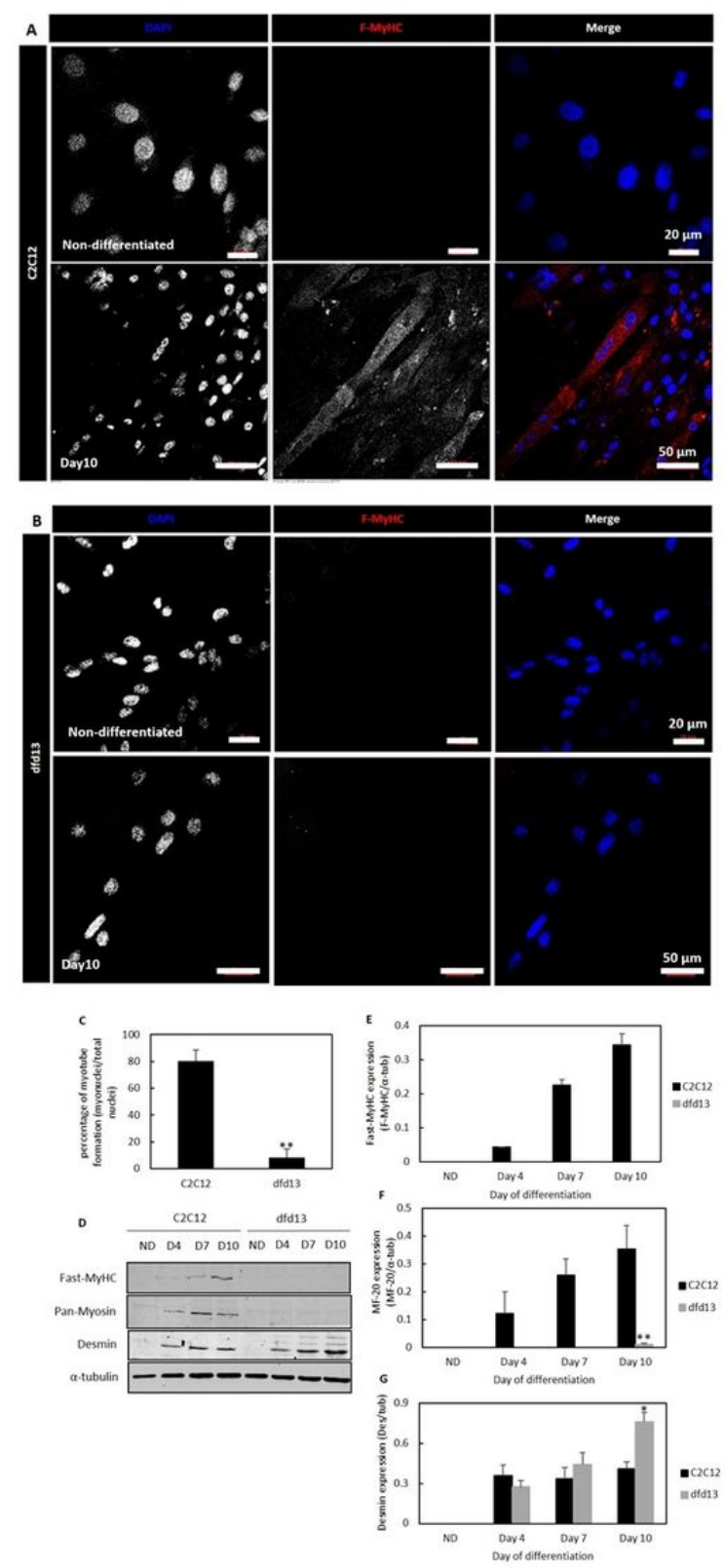

\section{Figure 1}

Dystrophin-deficient myoblasts have impaired differentiation capacity. Approximately 1.5 x 104 of both C2C12 (nondystrophic) and dfd13 (dystrophin-deficient) myoblasts were cultured in GM (on the acid-etched coverslip) before being transferred to DM and allowed to differentiate for 10. Immunofluorescence analysis of F-MyHC in (A) C2C12 and (B) dfd13 cells during the non-differentiated stage and after 10 days of differentiation. (C) Percentage myotube formation was calculated by counting the nuclei (DAPI) present in myotubes (myonuclei) per total nuclei from 10 random microscope fields. (D) Representative immunoblot of the proteins during myoblast differentiation with the a-tubulin expression as a loading control. Densitometry analyses of (E) F-MyHC (MY-32), (F) pan-myosin (MF20), and (G) desmin expression. The graphs represent an average of three repeats from different samples. All data are presented as mean \pm S.D. ND: non-differentiated; D10: day 10 of differentiation; **: significantly different $(p<0.01)$ compared to $\mathrm{C} 2 \mathrm{C} 12$ myoblasts. *: significantly different $(p<0.05)$ compared to C2C12 myoblasts GM: growth medium (DMEM + 10\% FCS); DM: differentiation medium (DMEM + 2\% horse serum). 

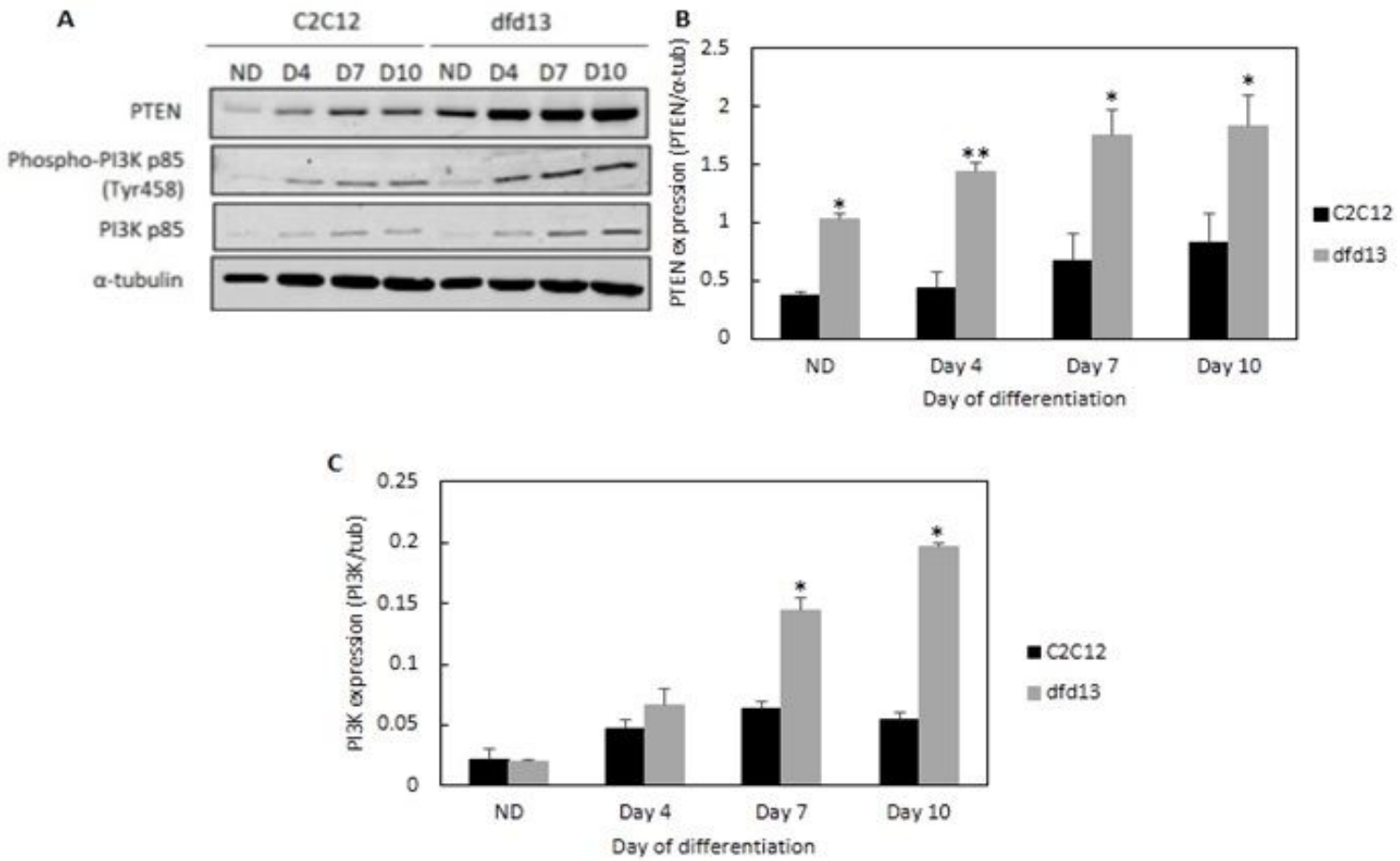

- $\mathrm{C} 2 \mathrm{C} 12$

II dfd13

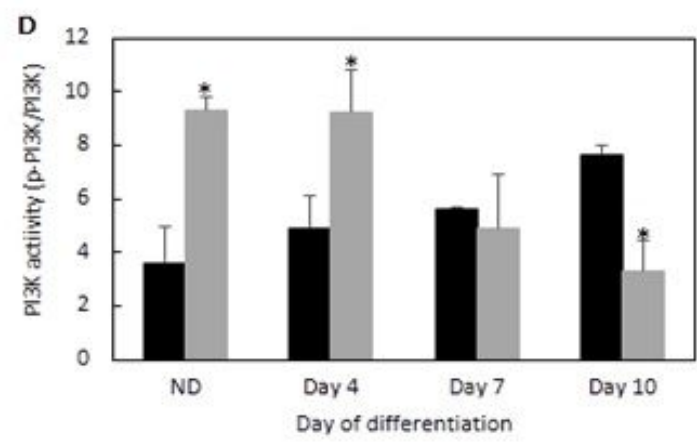

- $\mathrm{C} 2 \mathrm{C} 12$

IIf d 13

Figure 2

PTEN expression is higher while PI3K activation is decreased in dystrophin-deficient myoblasts. Myoblasts were cultured in GM until 80 to $90 \%$ confluent and culturing in DM for 10 days before total protein extraction and immunoblot analysis. (A) Immunoblot analysis of PTEN, phospho-PI3K (p85), and total PI3K during myoblasts differentiation with the a-tubulin expression as a loading control. Densitometry analysis of (B) PTEN expression, (C) total PI3K expression, and (D) PI3K activity. The graphs represent an average of three repeats from different samples. All data are presented as mean \pm S.D. 

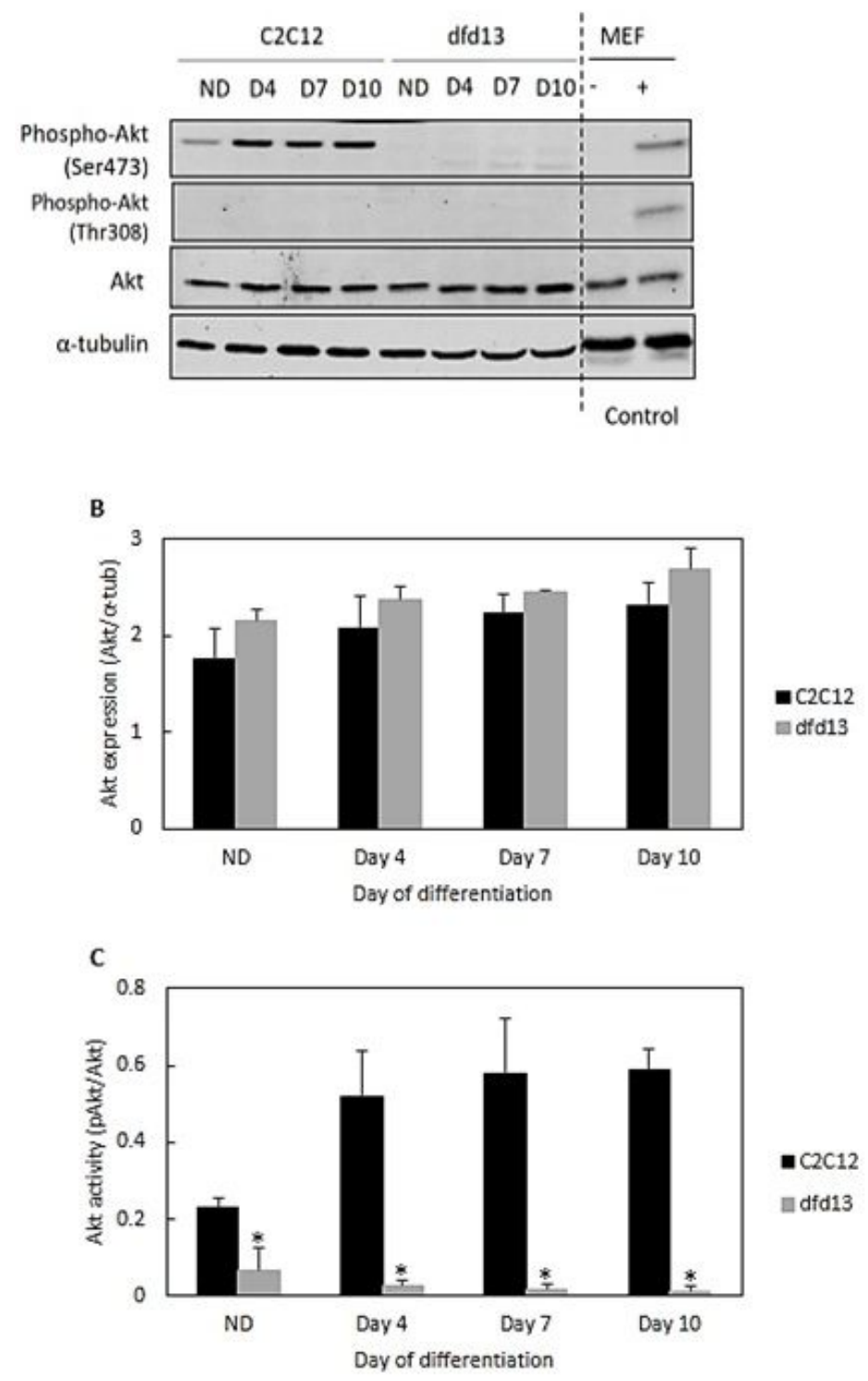

\section{Figure 3}

Akt is not/less activated in dystrophin-deficient myoblasts. Myoblasts were cultured in GM until 80 to $90 \%$ confluent and culturing in DM for 10 days before total protein extraction and immunoblot analysis. Untreated- and PDGF-treated MEF cells were used as a control for Akt phosphorylation at both Ser473 and Thr308. (A) Immunoblot analysis during myoblast differentiation with a-tubulin expression as a loading control. Densitometry analysis of (B) Akt expression and (C) Akt activation at Ser473. The graphs represent an average of three repeats from different samples. All data are presented as mean \pm S.D. 

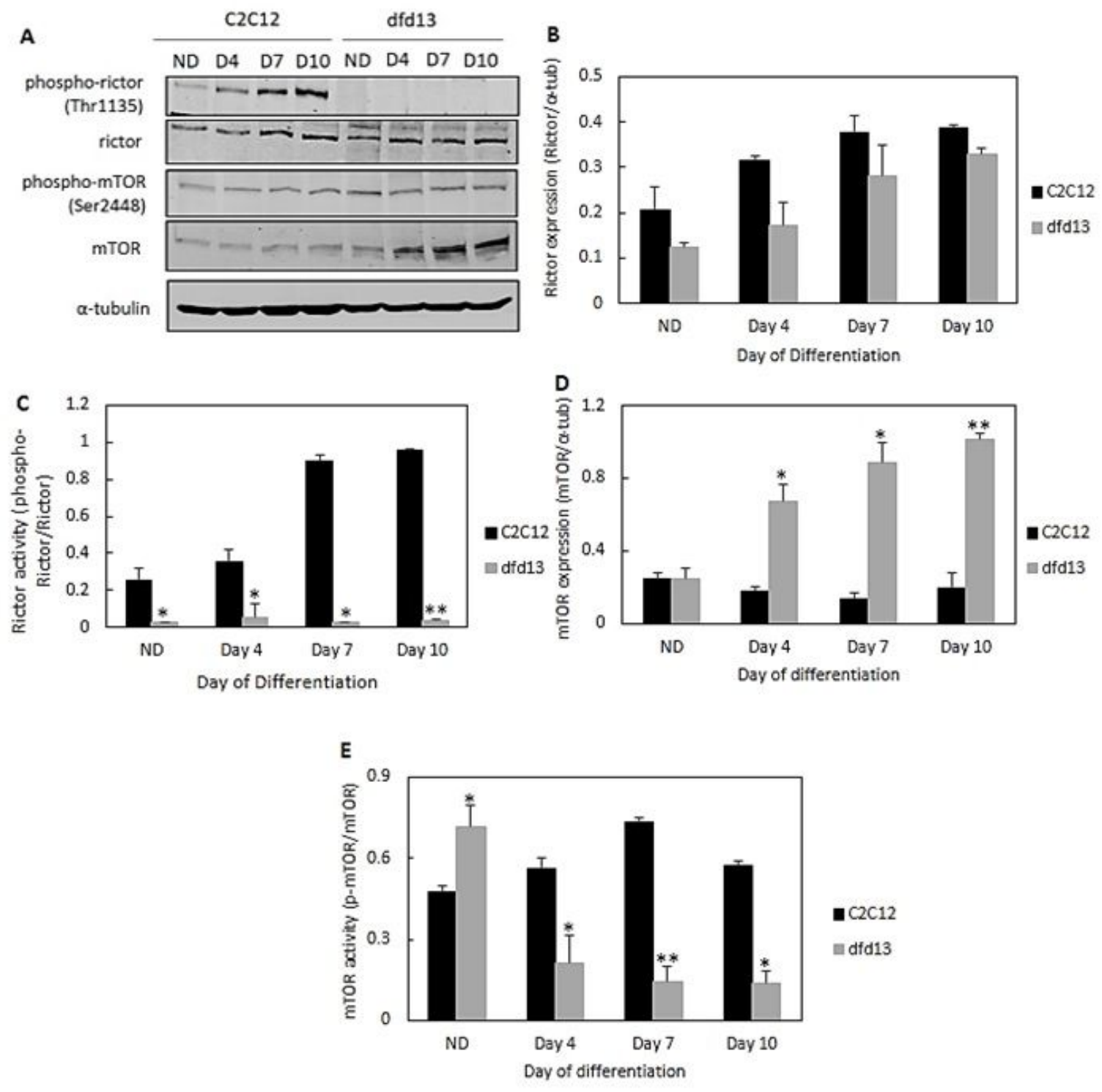

\section{Figure 4}

Rictor-mTORC2 activation is lower in dystrophin-deficient myoblasts. Myoblasts were cultured in GM until 80 to $90 \%$ confluent and culturing in DM for 10 days prior to total protein extraction and immunoblot analysis with antibodies recognizing phosphorictor (Thr1135), rictor, phospho-mTOR (Ser2448), and mTOR proteins. (A) Immunoblot analysis during myoblast differentiation with a-tubulin expression as a loading control. Densitometry analyses of (B) total rictor expression (C) rictor activity (D) total mTOR expression, and (E) mTOR activity. The graphs represent an average of three repeats from different samples. All data are presented as mean \pm S.D. 
A

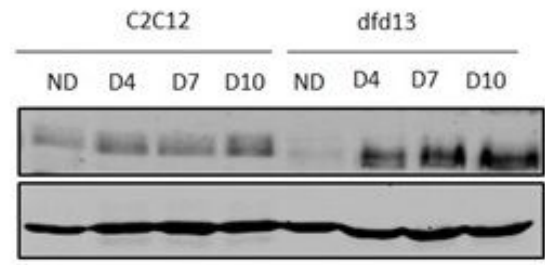

B

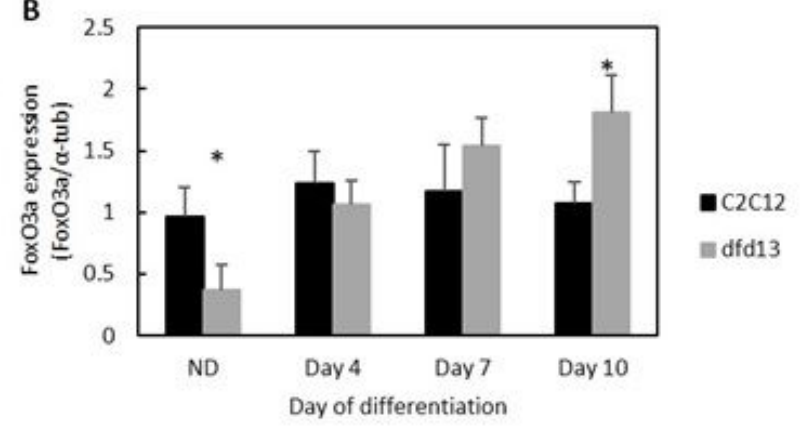

C
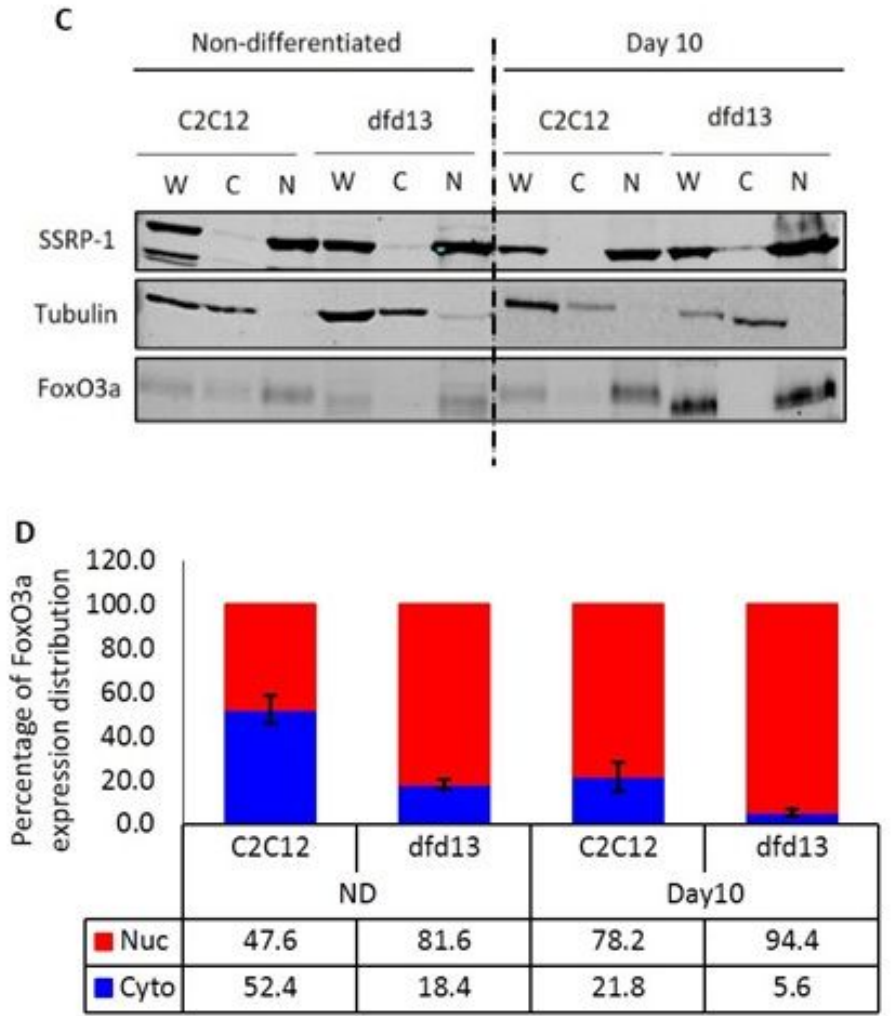

Figure 5

FoxO3a is highly increased and predominantly localized in differentiating dystrophin-deficient myoblasts. Myoblasts were cultured in GM until 80 to $90 \%$ confluent and culturing in DM for 10 days before total protein extraction and immunoblot analysis with antibodies recognizing FoxO3a. (A) Immunoblot analysis of proteins during myoblast differentiation with atubulin expression as a loading control. Densitometry analysis representing (B) FoxO3a expression. Subcellular protein extraction was performed at the indicated time points using the REAP protocol before immunoblotting with an antibody which recognizes FoxO3a. (C) Immunoblot of the proteins during myoblast differentiation with a-tubulin and SSRP1 expression as loading controls. Densitometry analysis representing (D) FoxO3a expression in the cytoplasm and nucleus. The graph represents an average of three repeats from different samples. All data are presented as mean \pm S.D. W: whole cell lysate; C: cytoplasm; N: nucleus. 

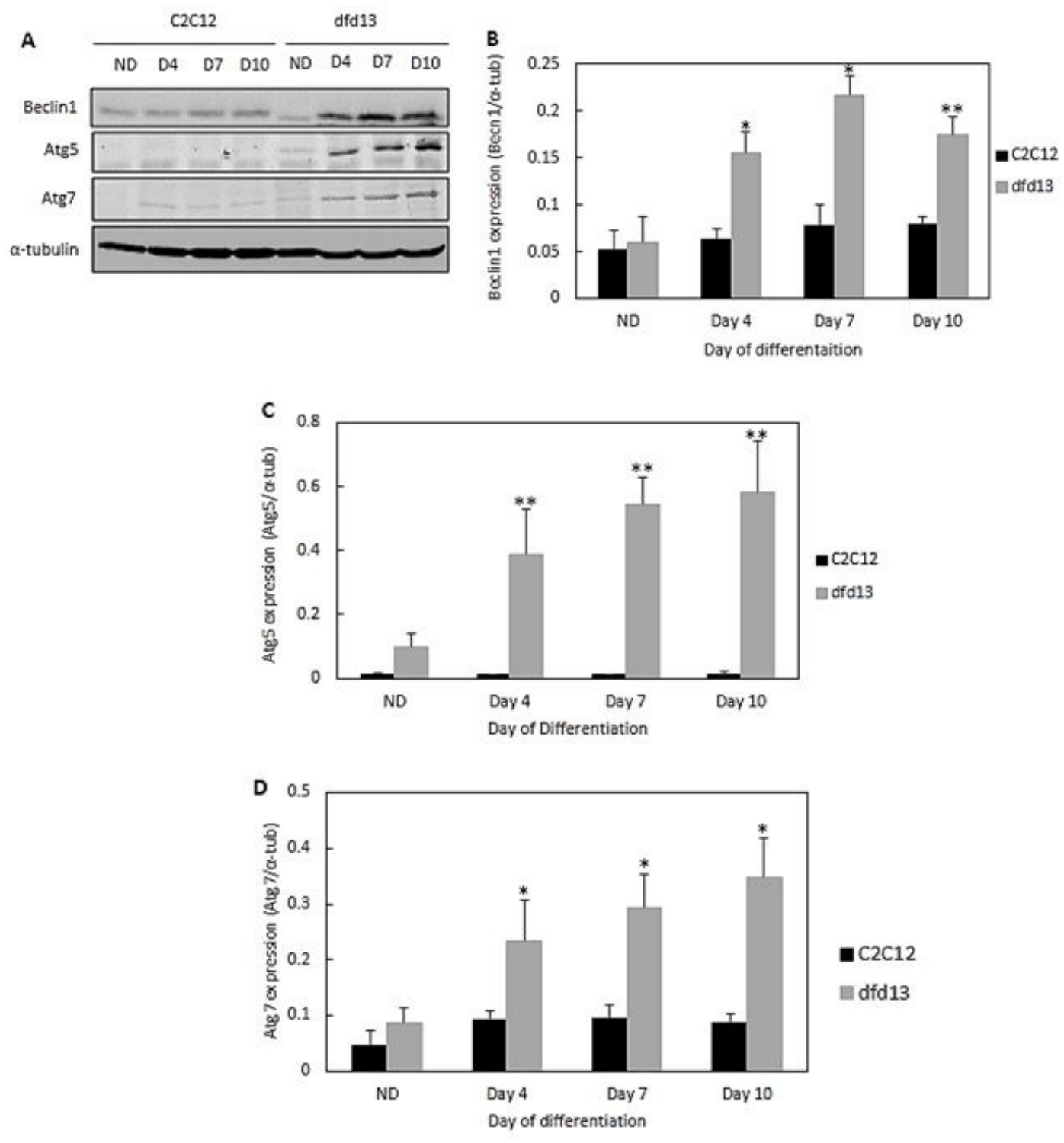

\section{Figure 6}

Expression of autophagy-related proteins is highly increased in differentiating dystrophin-deficient myoblasts. Myoblasts were cultured in GM until 80 to $90 \%$ confluent and culturing in DM for 10 days prior to total protein extraction and immunoblot analysis. (A) Immunoblot analysis of Beclin1, Atg5, and Atg7 expression with a-tubulin expression as a loading control. Densitometry analysis representing (B) Beclin1, (C) Atg5, and (D) Atg7 expression. The graphs represent an average of four repeats from different samples. All data are presented as mean \pm S.D. 

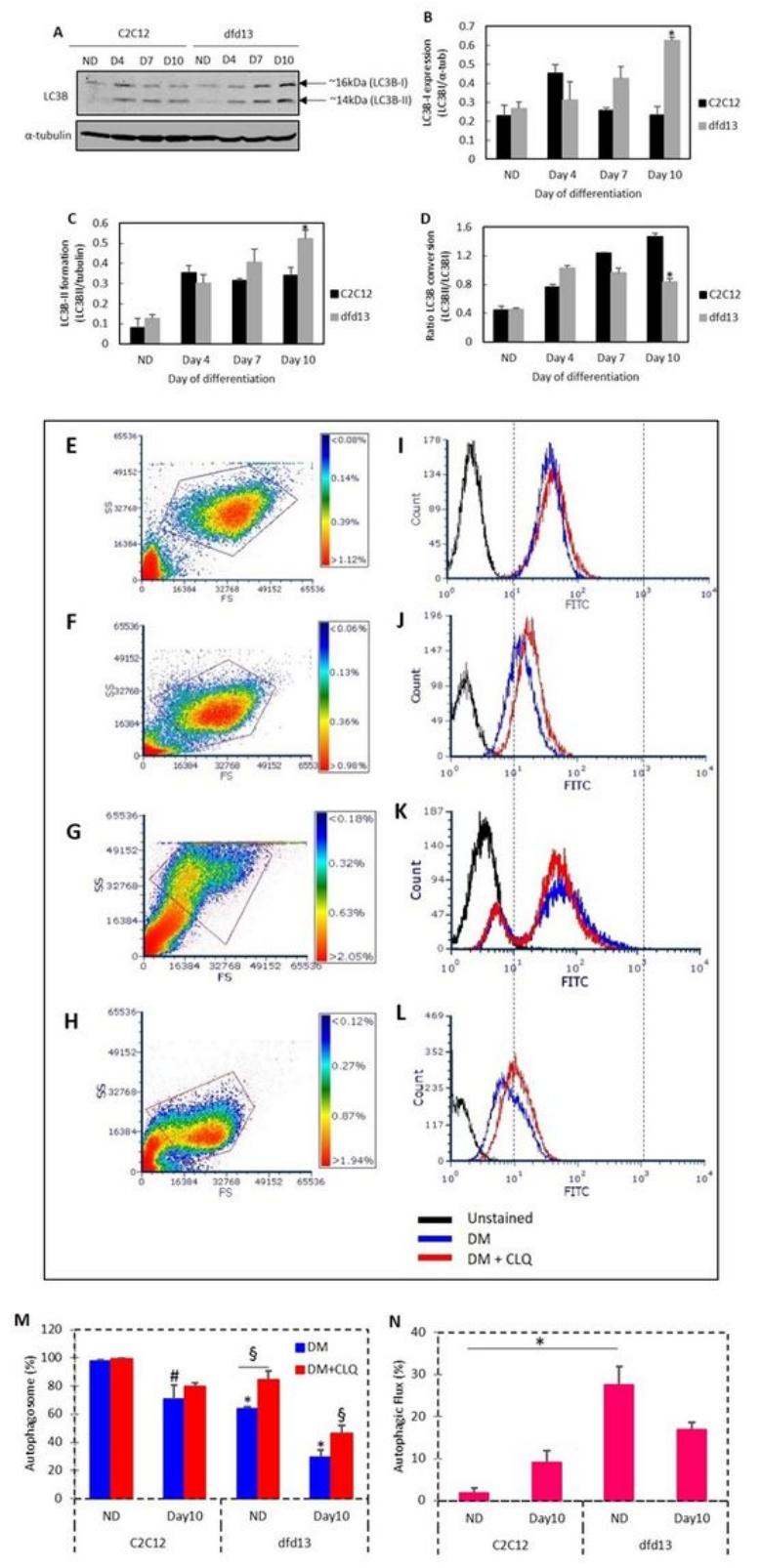

\section{Figure 7}

LC3B-II expression is increased but autophagic flux is decreased in differentiating dystrophin-deficient myoblasts. Myoblasts were cultured in GM until 80 to $90 \%$ confluent and culturing in DM for 10 days prior to total protein extraction and immunoblot analysis with antibodies recognizing LC3B with a-tubulin expression as a loading control. Densitometry analysis of (B) LC3B-I expression, (C) LC3B-II expression, and (D) the ratio of LC3B-II/LC3B-I. For flow cytometry analysis, three different conditions were set up for each stage: 1) unstained cells (negative control); 2) stained cells; and 3) stained cells with chloroquine treatment (positive control). Chloroquine treatment consisted of 4 hours' incubation at $37^{\circ} \mathrm{C}$. Cells were trypsinized and incubated with Cyto-ID Green stain solution before analysis using the FITC channel of a CyAn B flow cytometer (Beckman Coulter, USA). Data analysis was performed using FCS Express 6 Plus Research Edition De Novo Software, USA). The density plot images represent non-differentiated (E) C2C12 myoblasts, (F) dfd13 myoblasts; day10 differentiation (G) $\mathrm{C} 2 \mathrm{C} 12$ myoblasts and $(\mathrm{H})$ dfd13 myoblasts. The histogram overlays $(\mathrm{I}-\mathrm{L})$ represent each neighboring dot plot. $(\mathrm{M})$ The percentage of autophagosomes detected in myoblasts and $(\mathrm{N})$ percentage of autophagic flux within myoblasts. All bar graphs represent an average of three repeats from different samples. All data are presented as mean \pm S.D. Significantly different: * $(p<0.05)$ and $* *$ $(p<0.01)$ compared to C2C12 myoblasts. \# $(p<0.05)$ when compared with ND; $\S(p<0.05)$ when compared to DM+CLQ. 


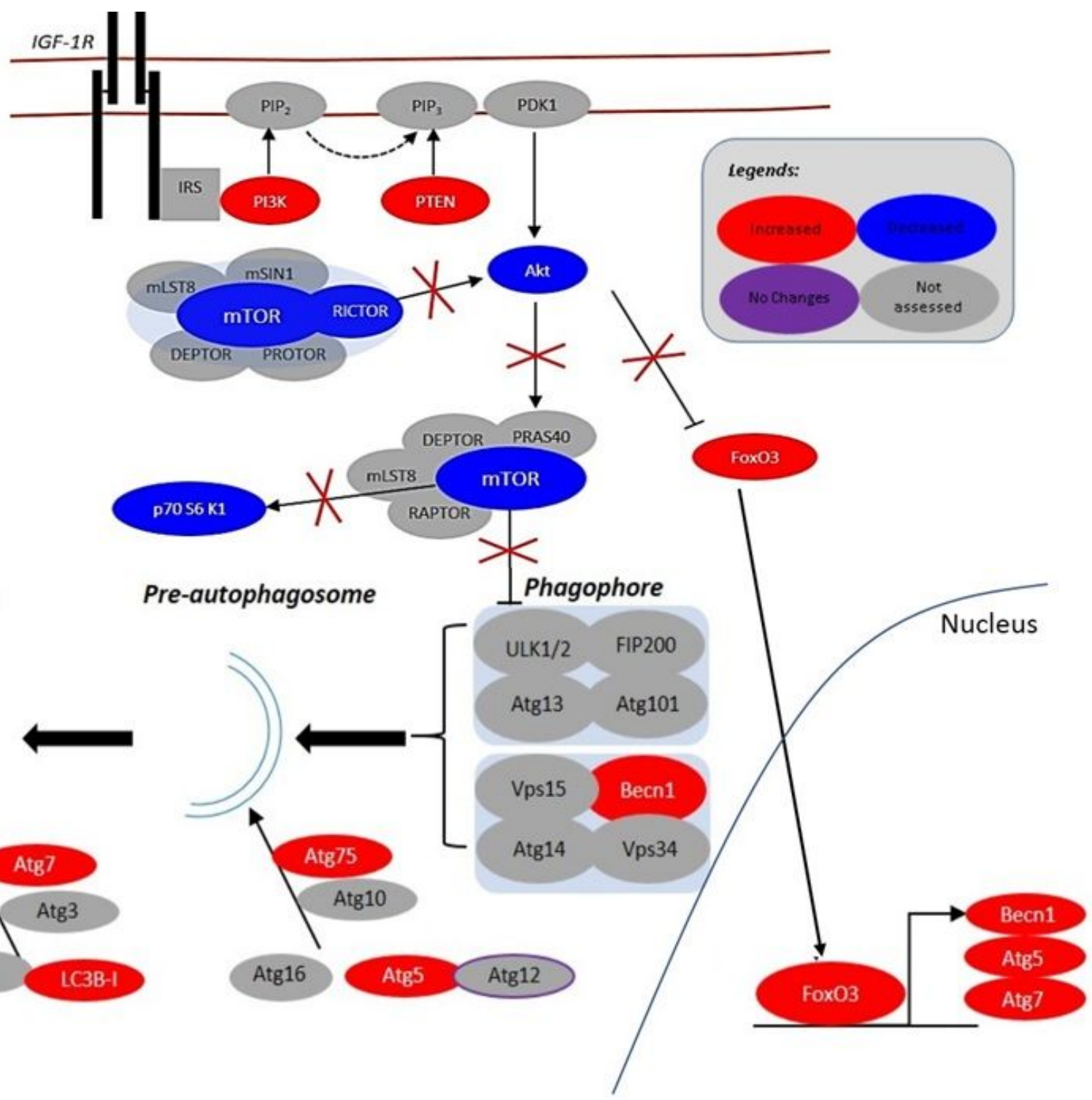

\section{Figure 8}

A Schematic of Autophagy modulation in dystrophin-deficient myoblasts. PTEN was found to be highly expressed in dystrophin-deficient myoblasts which leads to the reduction of activation of PI3K. Surprisingly, Akt was found not to be activated due to the inactivation of Rictor-mTORC2. Furthermore, downstream of Akt, i.e. p70S6K and FoxO3, there was defective activation. Activation of p70S6K was reduced, indicating that protein synthesis regulation is impaired. FoxO3 (unphosphorylated) expression accumulated thus increased FoxO3 nuclear translocation. Accumulation of translocated-FoxO3 to the nucleus increases the expression of autophagy related proteins, i.e. Atg5, Atg7, Beclin1. As a result, phagophore formation is increased and the maturation of autophagosome by Atgs; however, autophagic flux is reduced. The perturbation of $\mathrm{PI3K} /$ Akt increases nuclear-FoxO3, thus modulating excessive autophagosome formation while reducing autophagic flux in dystrophin-deficient myoblasts. 